TRANSACTIONS OF THE

AMERICAN MATHEMATICAL SOCIETY

Volume 355, Number 2, Pages 567-591

S 0002-9947(02)03099-4

Article electronically published on October 4, 2002

\title{
FORMULAS FOR TAMELY RAMIFIED SUPERCUSPIDAL CHARACTERS OF $\mathrm{GL}_{3}$
}

\author{
TETSUYA TAKAHASHI
}

\begin{abstract}
Let $F$ denote a $p$-adic local field of residual characteristic $p \neq 3$. This article gives formulas, valid on the regular elliptic set, for the irreducible supercuspidal characters of $\mathrm{GL}_{3}(F)$ which correspond to characters of a ramified Cartan subgroup. In the case in which $F$ does not contain cube roots of unity, i.e., the case in which ramified cubic extensions of degree 3 over $F$ cannot be Galois, base change results concerning "simple types" due to Bushnell and Henniart (1996) are used in the proofs.
\end{abstract}

\section{INTRODUCTION}

Let $F$ be a nonarchimedean local field of residual characteristic $p \neq 3$, let $A \mid F$ be a central simple algebra of reduced degree 3 , and let $E$ be a field such that $F \subsetneq E \subset A$. Then either $A=D$, a division algebra, or $A=\mathrm{M}_{3}(F)$, the algebra of all $3 \times 3$ matrices. Moreover, every compact mod center Cartan subgroup of $A^{\times}$is of the form $E^{\times}$for some such $E$, and every irreducible supercuspidal representation of $A^{\times}$corresponds to a quasi-character of some such $E^{\times}$([16], [6]).

In this paper we calculate formulas, valid on the regular elliptic set, for the supercuspidal characters of $A^{\times}$which correspond to characters of $E^{\times}$for ramified $E$. The reader may consult [19], in which we have discussed the case $E \mid F$ unramified, and 20, which deals with the more complicated case for $\mathrm{GL}_{2}(F)$ in which $E$ is a wildly ramified field, i.e. $p=2$. We do not give character values on the split torus here (see 17] for results pertaining to this problem). We mention that the character values on the regular elliptic set are sufficient to uniquely determine a supercuspidal representation (or, more generally, an arbitrary discrete series representation).

Let $D_{n} \mid F$ be a division algebra of index $n$. The "abstract matching theorem" of Badulescu 22, Deligne-Kazhdan-Vigneras 8], and Rogawski [18] implies the existence of a bijection between the set of irreducible representations of $D_{n}^{\times}$and the set of essentially square-integrable representations of $\mathrm{GL}_{n}(F)$ which preserves the characters on the corresponding regular elliptic sets up to the sign $(-1)^{n-1}$ (cf. Theorem 1.5 below). In the tame case (i.e. $(p, n)=1$ ) Moy [16] proved the existence of a bijection between these sets of representations which respects the concrete construction of the representations by Howe [12. In general, the relationship between these two bijections is unknown, but in the case $n$ is a prime $\neq p$ it is known that the two bijections coincide ([10]). Therefore, it suffices to determine the character

Received by the editors September 28, 1998 and, in revised form, May 2, 2002.

2000 Mathematics Subject Classification. Primary 22E50, Secondary 11F70.

Key words and phrases. Characters, supercuspidal, base change.

(C)2002 American Mathematical Society 
formula on either $D_{3}^{\times}$or $\mathrm{GL}_{3}(F)$ and to use the Howe-Moy bijection to obtain the formula for the corresponding representation on the other group (cf. Theorem 1.5).

Let $l$ be an odd prime and let $L \mid F$ be an unramified extension of degree $l$. In [19] we gave the character formulas of $\mathrm{GL}_{l}(F)$ and $D_{l}^{\times}$which correspond to a quasicharacter of $L^{\times}$(see [13] for the case $l=2$ ). To obtain these results we used the fact that $L \mid F$ is Galois. In the present instance we have to treat the case in which $E \mid F$ is not Galois, and we use the base change results of Bushnell and Henniart, which concern Bushnell-Kutzko "simple characters", to transfer our results from the case $E \mid F$ Galois to the non-Galois case. Since the Bushnell-Henniart results apply only to the split case, we work with $\mathrm{GL}_{3}(F)$ and use Howe-Moy theory to deduce the corresponding $D_{3}^{\times}$results; it is also easier to determine our character formulas near the conductor on $\mathrm{GL}_{3}(F)$ than on $D_{3}^{\times}$(compare our Lemma 2.10 to the proof of Theorem 1.10 in [13]). Our main results are Theorems 2.13 and 3.9.

Let us summarize the contents of this paper, indicating its organization:

Section 1 reviews the construction of irreducible supercuspidal representations $\pi_{\theta}$ of $\mathrm{GL}_{3}(F)$ (of representations $\pi_{\theta}^{\prime}$ of $D^{\times}$) from generic (see Definition 1.1) quasicharacters $\theta$ of $E^{\times}$. Note that $\pi_{\theta}$ is not always monomial; in the contrary case we represent its character as a $\mathbb{Q}$-linear combination of monomial characters.

Section 2 deals with the computation of the character of $\pi_{\theta}$ when $E \mid F$ is Galois. We represent $\pi_{\theta}$ as $\operatorname{ind}_{B}^{\mathrm{GL}_{3}(F)} \kappa_{\theta}$, where $B$ is the normalizer of an Iwahori subgroup of $\mathrm{GL}_{3}(F)$ and $\kappa_{\theta}$ is an irreducible representation of $B$ ("ind" denotes compact induction). We decompose the restriction $\left.\kappa_{\theta}\right|_{E \times}$ and use Mackey theory to calculate the characters of the constituents. Our reasoning here is reminiscent of 1.2 in 19; we obtain the character formula except near the conductor. The rest of the formula comes directly from the explicit representation of $\left.\kappa_{\theta}\right|_{E \times}$ (see Lemma 2.10). This is the only new idea in the section. Results of Kutzko [15] imply that the character of $\kappa_{\theta}$ equals the character of $\pi_{\theta}$ on the elliptic set.

Section 3 treats the case in which $E \mid F$ is not Galois. In this case, we consider an unramified quadratic extension $L \mid F ; L$ contains cube roots of unity, $E L \mid L$ is Galois, and both the methods and results of Section 2 apply to $\mathrm{GL}_{3}(L)$. By Bushnell and Henniart [4] we have a base change lift $\kappa_{L}$ of $\left.\kappa_{\theta}\right|_{B^{1}}$ to $B_{L}^{1}$ such that the twisted trace of $\kappa_{L}$ by a generator $\tau$ of $\operatorname{Gal}(L \mid F)$ gives the trace of $\kappa_{\theta}$ (see Proposition 3.3, and see above Proposition 3.3 for the notations $B^{1}$ and $B_{L}^{1}$ ). Note that we do not have to assume that the characteristic of $F$ is zero, since we do not use the Arthur-Clozel base change lift (1]). We calculate the twisted trace of $\kappa_{L}$ much as in Section 2; we state our main result as Theorem 3.9. This is the interesting application of the Bushnell-Henniart base change lift.

Our final character formulas seem simple and beautiful. As in the unramified case, the analog of Weyl's character formula holds. By contrast we know that the formulas for wildly ramified characters are more complicated (see [20]).

To conclude this introduction, we compare our formula with the result of [7]. The same type of character formula for the division algebra of reduced degree $l$ was given by Corwin, Moy and Sally, Jr. 7]. Their character formula contains some Gauss sum associated with a quadratic form. They have only shown that this Gauss sum is a fourth root of unity when $p \neq 2$. In this paper, we treat only the case $l=3$, but we have determined the root number completely, including the case $p=2$ in sections 2 and 3. Moreover we find that the Kloosterman sum appears in the character formula (cf. Lemma 2.10). This simplifies Theorem 4.1 (d) in [7]. 
Notation. Let $F$ be a nonarchimedean local field. We denote by $\mathcal{O}_{F}, P_{F}, \varpi_{F}, k_{F}$ and $v_{F}$ the maximal order of $F$, the maximal ideal of $\mathcal{O}_{F}$, a prime element of $P_{F}$, the residue field of $F$ and the valuation of $F$ normalized by $v_{F}\left(\varpi_{F}\right)=1$. Let $q=\left|k_{F}\right|$. When $A$ is a division algebra over $F$, the reduced trace of $A$ to $F$ is denoted Trd. The usual matrix trace is denoted by Tr. Hereafter we fix an additive character $\psi$ of $F$ whose conductor is $P_{F}$, i.e., $\psi$ is trivial on $P_{F}$ and not trivial on $\mathcal{O}_{F}$. For an extension $E$ over $F$, we denote by $\operatorname{tr}_{E}, n_{E}$ the trace and norm to $F$ respectively. We set $\psi_{E}=\psi \circ \operatorname{tr}_{E}$. For an irreducible admissible representation $\pi$ of $A^{\times}$, the conductoral exponent of $\pi$ is defined to be the integer $f(\pi)$ such that the local constant $\varepsilon(s, \pi, \psi)$ of Godement and Jacquet [9] is of the form $a q^{-s(f(\pi)-3)}$. We call $\pi$ minimal if

$$
f(\pi)=f_{\min }(\pi)=\min _{\eta} f(\pi \otimes(\eta \circ \mathrm{Nr})),
$$

where $\eta$ runs through the set of quasi-characters of $F^{\times}$. Let $G$ be a totally disconnected, locally compact group. We denote by $\widehat{G}$ the set of (equivalence classes of) irreducible admissible representations of $G$. For a closed subgroup $H$ of $G$ and a representation $\rho$ of $H$, we denote by $\operatorname{Ind}_{H}^{G} \rho\left(\operatorname{resp} . \operatorname{ind}_{H}^{G} \rho\right)$ the induced representation (resp. compactly induced representation) of $\rho$ to $G$. For a representation $\pi$ of $G$, we denote by $\left.\pi\right|_{H}$ the restriction of $\pi$ to $H$.

\section{Construction of the Representation}

Let $E$ be a ramified extension of $F$ of degree 3. $E$ can be embedded in $A$, and the embedding is unique up to conjugacy. In this section, we recall the construction of the supercuspidal representation of $A^{\times}$from the quasi-character of $E^{\times}$.

Definition 1.1. Let $\theta$ be a quasi-character of $E^{\times}$and $f(\theta)=\min \{n \mid \operatorname{Ker} \theta \supset$ $\left.1+P_{E}^{n}\right\} . \theta$ is called generic if $f(\theta) \not \equiv 1 \bmod 3$.

We write $\widehat{E}^{\times}$gen for the set of generic quasi-characters of $E^{\times}$. If $\theta \in \widehat{E}_{\text {gen }}$, then there exists $\gamma_{\theta} \in P_{E}^{1-f(\theta)}-P_{E}^{2-f(\theta)}$ such that $F\left(\gamma_{\theta}\right)=E$ and

$$
\theta(1+x)=\psi\left(\operatorname{tr}_{E \mid F}\left(\gamma_{\theta} x\right)\right) \text { for all } \quad x \in P_{E}^{[(f(\theta)+1) / 2]} .
$$

Let us begin the construction of an irreducible supercuspidal representation of $A^{\times}=\mathrm{GL}_{3}(F)$ corresponding to a generic character of $E^{\times}$. We choose $\theta \in \widehat{E}_{\text {gen }}$ such that $f(\theta)=n+1 \not \equiv 1$. We assume that the prime element $\varpi_{E} \in E$ satisfies $\varpi_{E}^{3}=\varpi_{F}$; using the $F$-basis $\left\{\varpi_{E}^{2}, \varpi_{E}, 1\right\}$ of $E$, we identify $A$ with $\operatorname{End}_{F}(E)$ and $G=\mathrm{GL}_{3}(F)$ with $\operatorname{Aut}_{F}(E)$. Using the lattice flag $\left\{P_{E}^{i}\right\}_{i \in \mathbb{Z}}$, we construct the maximal compact modulo center subgroup which contains $E^{\times}$:

Definition 1.2. For $i \in \mathbb{Z}$, set $A^{i}=\left\{f \in \mathrm{M}_{3}(F) \mid f\left(P_{E}^{j}\right) \subset P_{E}^{j+i}\right.$ for all $\left.j \in \mathbb{Z}\right\}$. Put $K=\left(A^{0}\right)^{\times}, B=E^{\times} K$ and $K^{i}=1+A^{i}$ for $i \geq 1$.

We note that $K$ is an Iwahori subgroup of $\mathrm{GL}_{3}(F)$ and $B$ is its normalizer. At the first step toward the construction of a supercuspidal character of $G$ from $\theta$, we construct a representation of $B$. We have $\gamma=\gamma_{\theta} \in P_{E}^{-n}$ such that $\theta(1+x)=\psi_{E}(\gamma x)$ for all $x \in P_{E}^{m}(m=[(n+2) / 2])$; we define a character $\psi_{\gamma}$ of $K^{m}$ by setting $\psi_{\gamma}(1+x)=\psi(\operatorname{Trd}(\gamma x))$ for $x \in A^{m}$. Set $H=E^{\times} K^{m}$ and define a quasi-character $\rho_{\theta}$ of $H$ by setting

$$
\rho_{\theta}(h \cdot g)=\theta(h) \psi_{\gamma}(g) \quad \text { for } \quad h \in E^{\times}, \quad g \in K^{m} .
$$


Let $J$ denote the stabilizer of $\psi_{\gamma}$ in $B$, i.e.,

$$
J=\left\{a \in B \mid \psi_{\gamma}\left(a x a^{-1}\right)=\psi_{\gamma}(x) \text { for } x \in K^{m}\right\} .
$$

Then $J=E^{\times} K^{m^{\prime}}$, where $m^{\prime}=[(n+1) / 2]$.

If $n+1=2 m$ is even, then $J=H=E^{\times} K^{m}$. By Clifford theory, $\operatorname{Ind}_{H}^{B} \rho_{\theta}$ is, in this case, an irreducible representation of $B$. We set

$$
\kappa_{\theta}=\operatorname{Ind}_{H}^{B} \rho_{\theta} .
$$

If $n+1=2 m-1$ is odd, then $J=E^{\times} K^{m-1}$; so we have to determine an irreducible component of $\operatorname{Ind}_{H}^{J} \rho_{\theta}$. For a subgroup $M \subset B$, we write $M^{1}=M \cup$ $F^{\times} K$. In particular, $H^{1}=F^{\times}\left(1+P_{E}\right) K^{m}$. It is known and not difficult to show that

$$
\left.\left(\operatorname{Ind}_{H}^{J} \rho_{\theta}\right)\right|_{J^{1}}=\operatorname{Ind}_{H^{1}}^{J^{1}}\left(\left.\rho_{\theta}\right|_{H_{1}}\right)=q \eta .
$$

This $\eta$ can be extended to $J$ in $\left|E^{\times} / F^{\times}\left(1+P_{E}\right)\right|=3$ ways. To determine the extension by $\theta$, we will express it by a linear combination of $\operatorname{Ind}_{H}^{J} \rho_{\theta \otimes \chi}(\chi \in$ $\left.\left(E^{\times} / F^{\times}\left(1+P_{E}\right)\right)^{\wedge}\right)$.

Lemma 1.3. Define the virtual representation $\kappa_{\theta}$ of $J$ by

$$
\kappa_{\theta}=\left\{\begin{array}{l}
\frac{1-q}{3 q} \sum_{\chi \in\left(E^{\times} / F^{\times}\left(1+P_{E}\right)\right)^{\wedge}} \operatorname{Ind}_{H}^{B} \rho_{\theta \otimes \chi}+\operatorname{Ind}_{H}^{B} \rho_{\theta} \quad \text { if } q \equiv 1 \quad \bmod 3, \\
\frac{1+q}{3 q} \sum_{\chi \in\left(E^{\times} / F^{\times}\left(1+P_{E}\right)\right)^{\wedge}} \operatorname{Ind}_{H}^{B} \rho_{\theta \otimes \chi}-\operatorname{Ind}_{H}^{B} \rho_{\theta} \quad \text { if } q \equiv 2 \quad \bmod 3 .
\end{array}\right.
$$

Then $\kappa_{\theta}$ is a real representation and an irreducible component of $\operatorname{Ind}_{H}^{B} \rho_{\theta}$.

Proof. Let $\left\{\eta_{1}, \eta_{2}, \eta_{3}\right\}$ be the set of the extensions of $\eta$ to $J$ and $\left(E^{\times} / F^{\times}\left(1+P_{E}\right)\right)^{\wedge}=$ $\left\{\chi_{1}, \chi_{2}, \chi_{3}\right\}$. It follows from Lemma 3.5.35 in [16] that

$$
\operatorname{Ind}_{H}^{J} \rho_{\theta}=\frac{\left(q-\left(\frac{q}{3}\right)\right)}{3} \sum_{i=1}^{3} \eta_{i}+\left(\frac{q}{3}\right) \eta_{j}
$$

for a unique $j$, where $\left(\frac{q}{3}\right)$ is the Legendre symbol. Let us denote $\eta_{\theta}$ by this $\eta_{j}$. From this irreducible decomposition of $\operatorname{Ind}_{H}^{J} \rho_{\theta}$, we have

$$
\left(\begin{array}{l}
\operatorname{Ind}_{H}^{J} \rho_{\theta \otimes \chi_{1}} \\
\operatorname{Ind}_{H}^{J} \rho_{\theta \otimes \chi_{2}} \\
\operatorname{Ind}_{H}^{J} \rho_{\theta \otimes \chi_{3}}
\end{array}\right)=\left(\left(\frac{q}{3}\right)\left(\begin{array}{lll}
1 & 0 & 0 \\
0 & 1 & 0 \\
0 & 0 & 1
\end{array}\right)+\frac{\left(q-\left(\frac{q}{3}\right)\right)}{3}\left(\begin{array}{lll}
1 & 1 & 1 \\
1 & 1 & 1 \\
1 & 1 & 1
\end{array}\right)\right)\left(\begin{array}{l}
\eta_{\theta \otimes \chi_{1}} \\
\eta_{\theta \otimes \chi_{2}} \\
\eta_{\theta \otimes \chi_{3}}
\end{array}\right) .
$$

By the Clifford theory, $\kappa_{\theta}=\operatorname{Ind}_{J}^{B} \rho_{\theta}$ is an irreducible representation of $B$. Thus we obtain the desired formula for $\kappa_{\theta}$ by calculating the inverse of the coefficient matrix.

The following result is well-known. (See [16.)

Theorem 1.4. Let the notation be as above. Then $\kappa_{\theta}$ is an irreducible representation of $B$. Put $\pi_{\theta}=\operatorname{ind}_{B}^{G} \kappa_{\theta}$. Then $\pi_{\theta}$ is an irreducible supercuspidal representation of $G$ such that:

1. the L-function of $\pi_{\theta}$ is 1 ;

2. $\varepsilon\left(\pi_{\theta}, \psi\right)=\varepsilon\left(\theta, \psi_{E}\right)$; in particular $f\left(\pi_{\theta}\right)=f(\theta)+3$.

Any irreducible supercuspidal representation $\pi$ of $G$ such that $f(\pi) \not \equiv 0 \bmod 3$ can be written in the form $\pi=\pi_{\theta}$ for some $\theta \in \widehat{E}_{\mathrm{gen}}^{\times}$, where $E$ is a ramified extension of degree 3 over $F$. 
Next we construct an irreducible representation of $D^{\times}$from $\theta \in E_{\text {gen }}^{\times}$. Let $f(\theta)=n+1$. We recall that $n \not \equiv 0 \bmod 3$. We define a function $\psi_{\gamma}$ on $1+P_{D}^{m}$ by $\psi_{\gamma}(1+x)=\psi(\operatorname{Trd}(\gamma x))$ for $x \in P_{D}^{m}$. Then $\psi_{\gamma}$ is a quasi-character of $1+P_{D}^{m}$. Set $H^{\prime}=E^{\times}\left(1+P_{D}^{m}\right) \subset D^{\times}$and define a quasi-character $\rho_{\theta}^{\prime}$ of $H^{\prime}$ by

$$
\rho_{\theta}^{\prime}(h \cdot g)=\theta(h) \psi_{\gamma}(g) \quad \text { for } \quad h \in E^{\times}, \quad g \in 1+P_{D}^{m} .
$$

When $n+1$ is even, i.e. $n+1=2 m$, we set

$$
\pi_{\theta}^{\prime}=\operatorname{Ind}_{H^{\prime}}^{D^{\times}} \rho_{\theta}^{\prime}
$$

When $n+1$ is odd, i.e. $n+1=2 m-1$, $\operatorname{Ind}_{H^{\prime}}^{D^{\times}} \rho_{\theta}^{\prime}$ is not irreducible. As in the $\mathrm{GL}_{3}$ case, we can take its irreducible component as a $\mathbb{Q}$-linear combination of $\operatorname{Ind}_{H^{\prime}}^{D^{\times}} \rho_{\theta \otimes \chi}^{\prime}$ $\left(\chi \in\left(E^{\times} / F^{\times}\left(1+P_{E}\right)\right)^{\wedge}\right)$.

Lemma 1.5. Define the virtual representation $\pi_{\theta}^{\prime}$ of $D^{\times}$by

$$
\pi_{\theta}^{\prime}=\left\{\begin{array}{l}
\frac{1-q}{3 q} \sum_{\chi \in\left(E^{\times} / F^{\times}\left(1+P_{E}\right)\right)^{\wedge}} \operatorname{Ind}_{H^{\prime}}^{D^{\times}} \rho_{\theta \otimes \chi}^{\prime}+\operatorname{Ind}_{H^{\prime}}^{D^{\times}} \rho_{\theta}^{\prime} \quad \text { if } q \equiv 1 \quad \bmod 3, \\
\frac{1+q}{3 q} \sum_{\chi \in\left(E^{\times} / F^{\times}\left(1+P_{E}\right)\right)^{\wedge}} \operatorname{Ind}_{H^{\prime}}^{D^{\times}} \rho_{\theta \otimes \chi}^{\prime}-\operatorname{Ind}_{H^{\prime}}^{D^{\times}} \rho_{\theta}^{\prime} \quad \text { if } q \equiv 2 \quad \bmod 3 .
\end{array}\right.
$$

Then $\pi_{\theta}^{\prime}$ is a real representation and an irreducible component of $\operatorname{Ind}_{H^{\prime}}^{D^{\times}} \rho_{\theta}^{\prime}$.

Proof. The argument from Lemma 3.5.28 to Lemma 3.5.35 in [16] can be applied to the division algebra case. Therefore we can prove this lemma in the same way as Lemma1.3.

The following result is essentially well-known. (See [3] and [16].)

Theorem 1.6. Let the notation be as above. Then $\pi_{\theta}^{\prime}$ is an irreducible minimal representation of $D^{\times}$such that:

1. the L-function of $\pi_{\theta}^{\prime}$ is 1 ;

2. $\varepsilon\left(\pi_{\theta}^{\prime}, \psi\right)=\varepsilon\left(\theta, \psi_{E}\right)$; in particular $f\left(\pi_{\theta}^{\prime}\right)=f(\theta)+3$.

Any irreducible representation $\pi^{\prime}$ of $D^{\times}$such that $f\left(\pi^{\prime}\right) \not \equiv 0 \bmod 3$ can be written in the form $\pi^{\prime}=\pi_{\theta}^{\prime}$ for some $\theta \in \widehat{E}_{\mathrm{gen}}^{\times}$, where $E$ is a ramified extension of degree 3 over $F$.

Now we define the correspondence $\pi_{\theta}^{\prime} \leftrightarrow \pi_{\theta}$ by parameterizing in each case the set of representations by the set of generic quasi-characters of $E^{\times}$(Howe's bijection [16]).

Proposition 1.7. The characters of $\pi_{\theta}$ and $\pi_{\theta}^{\prime}$ are equal on regular elliptic conjugacy classes, which are identified between the groups $D^{\times}$and $\mathrm{GL}_{3}(F)$.

Remark. The set of irreducible supercuspidal representations of $A^{\times}$with minimal conductor $\equiv 0 \bmod 3$ is parameterized by the set of regular quasi-characters of $L^{\times}$, where $L$ is an unramified extension of $F$ of degree 3 . The character formula for such a representation on the regular elliptic set was given in [19].

In concluding this section we reformulate a result of Kutzko ([15]) to put it into the precise form in which we shall apply it: 
Theorem 1.8. Let $x$ be a regular elliptic element of $G$.

1. If $F(x) \mid F$ is ramified and $x \notin F^{\times}\left(1+P_{F(x)}^{n+1}\right)$, then

$$
\chi_{\pi_{\theta}}(x)=\chi_{\kappa_{\theta}}(x) \text {. }
$$

2. If $F(x) \mid F$ is unramified and $x \notin F^{\times}\left(1+P_{F(x)}^{[(n+3) / 3]}\right)$, then

$$
\chi_{\pi_{\theta}}(x)=0 .
$$

Proof. It is obtained by applying Proposition 5.5 in [15] to our case.

\section{Character formula for the Galois case}

In this section, we treat the case in which $F$ contains a primitive cube root $\zeta$ of unity. Since $p \neq 3$, we have $q=p^{f} \equiv 1 \bmod 3$; in this case all ramified extensions of degree 3 of $F$ are Galois. We shall calculate a formula only for the character of $\kappa_{\theta}$; by applying Theorem 1.8 we obtain from this calculation a formula for the character of $\pi_{\theta}$. As in the first section, we identify $\mathrm{M}_{3}(F)$ with $\operatorname{End}_{F}(E)$ by the $F$-basis $\left\{\varpi_{E}^{2}, \varpi_{E}, 1\right\}$ of $E$. Thus we get the explicit matrix forms of various objects:

$$
\begin{aligned}
\varpi_{E} & =\left(\begin{array}{ccc}
0 & 1 & 0 \\
0 & 0 & 1 \\
\varpi_{F} & 0 & 0
\end{array}\right), \\
E & =\left\{\left(\begin{array}{ccc}
a & b & c \\
c \varpi_{F} & a & b \\
b \varpi_{F} & c \varpi_{F} & a
\end{array}\right) \mid a, b, c \in F\right\}, \\
K & =\left\{\left(\begin{array}{lll}
a_{11} & a_{12} & a_{13} \\
a_{21} & a_{22} & a_{23} \\
a_{31} & a_{32} & a_{33}
\end{array}\right) \mid \begin{array}{ll}
a_{i j} \in \mathcal{O}_{F} & \text { if } i<j \\
a_{i i} \in \mathcal{O}_{F}^{\times} & \\
a_{i j} \in P_{F} & \text { if } i>j
\end{array}\right\}, \\
A^{0} & =\left\{\left(\begin{array}{lll}
a_{11} & a_{12} & a_{13} \\
a_{21} & a_{22} & a_{23} \\
a_{31} & a_{32} & a_{33}
\end{array}\right) \mid \begin{array}{ll}
a_{i j} \in \mathcal{O}_{F} & \text { if } i \leq j \\
a_{i j} \in P_{F} & \text { if } i>j
\end{array}\right\}, \\
A^{1} & =\left\{\left(\begin{array}{lll}
a_{11} & a_{12} & a_{13} \\
a_{21} & a_{22} & a_{23} \\
a_{31} & a_{32} & a_{33}
\end{array}\right) \mid \begin{array}{ll}
a_{i j} \in \mathcal{O}_{F} & \text { if } i<j \\
a_{i j} \in P_{F} & \text { if } i \geq j
\end{array}\right\} .
\end{aligned}
$$

Let $\sigma$ be the generator of $\operatorname{Gal}(E \mid F)$ such that ${ }^{\sigma} \varpi_{E}=\varpi_{E} \zeta$.

Lemma 2.1. Put

$$
\xi=\left(\begin{array}{ccc}
1 & 0 & 0 \\
0 & \zeta^{2} & 0 \\
0 & 0 & \zeta
\end{array}\right)
$$

Then $\xi$ satisfies $\xi^{3}=1$,

$$
\xi x \xi^{-1}={ }^{\sigma} x \quad \text { for any } \quad x \in E,
$$

and

$$
\begin{array}{ccccccc}
\mathrm{M}_{3}(F) & = & E & \oplus & E \xi & \oplus & E \xi^{2}, \\
A^{0} & = & \mathcal{O}_{E} & \oplus & \mathcal{O}_{E} \xi & \oplus & \mathcal{O}_{E} \xi^{2} \\
A^{1} & = & P_{E} & \oplus & P_{E} \xi & \oplus & P_{E} \xi^{2} \\
A^{2} & = & P_{E}^{2} & \oplus & P_{E}^{2} \xi & \oplus & P_{E}^{2} \xi^{2}
\end{array}
$$

Proof. This is obvious from the above explicit matrix forms of $\varpi_{E}$ and $A^{i}$. 
Let $\theta$ be a generic quasi-character of $E^{\times}$with $f(\theta)=n+1$. If $n$ is odd, then $n+1=2 m$ and $\kappa_{\theta}=\operatorname{Ind}_{H}^{B} \rho_{\theta}$, where $H=E^{\times}\left(1+A^{m}\right)$. By Mackey theory $([22$, Proposition 22),

$$
\left.\kappa_{\theta}\right|_{E^{\times}}=\bigoplus_{a \in H \backslash B / E^{\times}} \operatorname{Ind}_{a^{-1} \operatorname{Ha\cap E}^{\times}}{ }^{a} \rho_{\theta},
$$

where ${ }^{a} \rho_{\theta}(x)=\rho_{\theta}\left(a x a^{-1}\right)$ for $x \in a^{-1} H a \cap E^{\times}$. If $n$ is even, then $n+1=2 m-1$ and

$$
\begin{aligned}
&\left.\kappa_{\theta}\right|_{E^{\times}}=\frac{1-q}{3 q} \sum_{\chi \in\left(E^{\times} / F^{\times}\left(1+P_{E}\right)\right)^{\wedge}} \sum_{a \in H \backslash B / E^{\times}} \operatorname{Ind}_{a^{-1} H a \cap E^{\times}}^{E^{\times}} \rho_{\theta \otimes \chi} \\
&+\sum_{a \in H \backslash B / E^{\times}} \operatorname{Ind}_{a^{-1} H a \cap E^{\times}}{ }^{a} \rho_{\theta},
\end{aligned}
$$

since $q \equiv 1 \bmod 3$.

For simplicity, we fix a generic character $\theta$ of $E^{\times}$with $f(\theta)=n+1$ and drop the subscript $\theta$ in the representations determined by $\theta$, e.g. $\rho=\rho_{\theta}, \kappa=\kappa_{\theta}$ and so on.

First we give a complete system of representatives for the set of the double cosets $H \backslash B / E^{\times}$.

Lemma 2.2. 1. Let $a=1+\alpha_{1} \xi+\alpha_{2} \xi^{2}$ for $\alpha_{1}, \alpha_{2} \in \mathcal{O}_{E}$. Then $a \in K$ is equivalent to $1+\alpha_{1}^{3}+\alpha_{2}^{3}-3 \alpha_{1} \alpha_{2} \notin P_{E}$.

2. Let $a=1+\alpha_{1} \xi+\alpha_{2} \xi^{2}, b=1+\beta_{1} \xi+\beta_{2} \xi^{2} \in K$. Then $H a=H b$ if and only if $\alpha_{i}-\beta_{i} \in P_{E}^{m}$ for $i=1,2$.

Proof. 1. Since $a$ is obviously in $A^{0}$, it is only necessary to determine necessary and sufficient conditions such that the diagonal entries belong to $\mathcal{O}_{F}^{\times}$, i.e., necessary and sufficient conditions that the product of the diagonal entries belong to $\mathcal{O}_{F}^{\times}$. Using explicit representations for the diagonal entries, multiplying them and simplifying by using the relation $0=1+\zeta+\zeta^{2}$, the reader may verify the given condition.

2. By [2.6, $H a=H b$ implies that there exist $\gamma_{0} \in \mathcal{O}_{E}^{\times}$and $\gamma_{1}, \gamma_{2} \in P_{E}^{m}$ such that $b=\left(\sum_{i=0}^{2} \gamma_{i} \xi^{i}\right) a$. Since $A^{0}=\mathcal{O}_{E} \oplus \mathcal{O}_{E} \xi \oplus \mathcal{O}_{E} \xi^{2}$ and $\xi x={ }^{\sigma} x \xi$ for $x \in E$, we obtain

$$
\begin{gathered}
1=\gamma_{0}+\left(\gamma_{1}{ }^{\sigma} \alpha_{2}+\gamma_{2}^{\sigma^{2}} \alpha_{1}\right) \\
\beta_{1}-\alpha_{1}=\left(\gamma_{0}-1\right) \alpha_{1}+\gamma_{1}+\gamma_{2}^{\sigma^{2}} \alpha_{2} \\
\beta_{2}-\alpha_{2}=\left(\gamma_{0}-1\right) \alpha_{2}+\gamma_{2}+\gamma_{1}^{\sigma} \alpha_{1}
\end{gathered}
$$

Therefore we have $\gamma_{0} \in 1+P_{E}^{m}$ and $\beta_{i}-\alpha_{i} \in P_{E}^{m} \quad(i=1,2)$.

Conversely we assume $\beta_{i}-\alpha_{i} \in P_{E}^{m} \quad(i=1,2)$. Put $b a^{-1}=\sum_{i=0}^{2} \gamma_{i} \xi^{i}$. By eliminating $\gamma_{0}$ from the above three equations, we obtain

$$
\begin{aligned}
& \beta_{1}-\alpha_{1}=\left(1-\alpha_{1}{ }^{\sigma} \alpha_{2}\right) \gamma_{1}+\left({ }^{\sigma^{2}} \alpha_{2}-\alpha_{1}{ }^{\sigma^{2}} \alpha_{1}\right) \gamma_{2}, \\
& \beta_{2}-\alpha_{2}=\left({ }^{\sigma} \alpha_{1}-\alpha_{2}{ }^{\sigma} \alpha_{2}\right) \gamma_{1}+\left(1-{ }^{\sigma^{2}} \alpha_{1} \alpha_{2}\right) \gamma_{2} .
\end{aligned}
$$

Noting that $\alpha_{i}-\beta_{i} \in P_{E}^{m}$, we use Cramer's Rule to verify that $\gamma_{i} \in P_{E}^{m}(i=1,2)$, i.e., we check that the determinant of the coefficient matrix for the system of two equations in the variables $\gamma_{1}$ and $\gamma_{2}$ belongs to $\mathcal{O}_{E}^{\times}$. A routine calculation reveals that this determinant equals $\operatorname{det}(a)$, as given in Lemma 2.5] below; clearly, $\operatorname{det}(a) \in$ $\mathcal{O}_{E}^{\times}$. Since $\operatorname{det}(a)$ is the denominator for Cramer's Rule and $\alpha_{i}-\beta_{i} \in P_{E}^{m}(i=1,2)$ 
imply that the numerator belongs to $P_{E}^{m}$, we see that $\gamma_{1}, \gamma_{2} \in P_{E}^{m}$. From the three preceding equations we then see that $\gamma_{0} \in 1+P_{E}^{m}$ too.

In order to give a set of representatives for $H \backslash B / E^{\times}$we introduce some more notation. Put $\mathcal{O}_{E}^{(1)}=\operatorname{Ker} n_{E}$ and

$$
M=\left\{\left({ }^{\sigma} \alpha \alpha^{-1}, \sigma^{2} \alpha \alpha^{-1}\right) \mid \alpha \in E^{\times}\right\} \subset \mathcal{O}_{E}^{(1)} \times \mathcal{O}_{E}^{(1)} .
$$

For $0<\mu<m$, we set

$$
\begin{aligned}
I_{\mu, 1} & =\left(1+P_{E}^{m-\mu}\right) \times P_{E}^{m} \backslash \varpi_{E}^{\mu} \mathcal{O}_{E}^{\times} \times P_{E}^{\mu} / M, \\
I_{\mu, 2} & =P_{E}^{m} \times\left(1+P_{E}^{m-\mu}\right) \backslash P_{E}^{\mu+1} \times \varpi_{E}^{\mu} \mathcal{O}_{E}^{\times} / M, \\
J_{\mu, 1} & =\left(\varpi_{F}^{\mu} n_{E}\left(\mathcal{O}_{E}^{\times}\right) /\left(1+P_{F}^{[(m-\mu+2) / 3]}\right)\right) \times\left(P_{E}^{2 \mu} / P_{E}^{m+\mu}\right), \\
J_{\mu, 2} & =\left(P_{E}^{2 \mu+1} / P_{E}^{m+\mu}\right) \times\left(\varpi_{F}^{\mu} n_{E}\left(\mathcal{O}_{E}^{\times}\right) /\left(1+P_{F}^{[(m-\mu+2) / 3]}\right)\right) .
\end{aligned}
$$

When $\mu=0$, we set

$$
\begin{aligned}
& I_{0,1}=\left(1+P_{E}^{m}\right) \times P_{E}^{m} \backslash\left\{\left(\beta_{1}, \beta_{2}\right) \in \mathcal{O}_{E}^{\times} \times \mathcal{O}_{E} \mid 1+\beta_{1}^{3}+\beta_{2}^{3}-3 \beta_{1} \beta_{2} \notin P_{E}\right\} / M, \\
& I_{0,2}=P_{E}^{m} \times\left(1+P_{E}^{m}\right) \backslash\left\{\left(\beta_{1}, \beta_{2}\right) \in P_{E} \times \mathcal{O}_{E}^{\times} \mid 1+\beta_{2}^{3} \notin P_{E}\right\} / M, \\
& J_{0,1}=\left\{\left(\beta_{1}, \beta_{2}\right) \in\left(n_{E}\left(\mathcal{O}_{E}^{\times}\right) / 1+P_{F}^{[(m+2) / 3]}\right) \times\left(\mathcal{O}_{E} / P_{E}^{m}\right) \mid\right. \\
& \left.1+\beta_{1}+\beta_{1}^{-1} \beta_{2}^{3}-3 \beta_{2} \notin P_{E}\right\}, \\
& J_{0,2}=\left\{\left(\beta_{1}, \beta_{2}\right) \in\left(P_{E} / P_{E}^{m}\right) \times\left(n_{E}\left(\mathcal{O}_{E}^{\times}\right) /\left(1+P_{F}^{[(m+2) / 3]}\right)\right) \mid 1+\beta_{2} \notin P_{E}\right\} .
\end{aligned}
$$

For $0 \leq \mu<m$, we set

$$
\tilde{I}_{\mu, i}=\left\{1+\beta_{1} \xi+\beta_{2} \xi^{2} \mid\left(\beta_{1}, \beta_{2}\right) \in I_{\mu, i}\right\} .
$$

Lemma 2.3. 1. A complete set of representatives of the double coset $H \backslash B / E^{\times}$is given by

$$
\begin{aligned}
\left\{1, \xi, \xi^{2}\right\} & \cup \bigcup_{\mu=0}^{m-1}\left(\tilde{I}_{\mu, 1} \cup \tilde{I}_{\mu, 2}\right) \cup \bigcup_{\mu=1}^{m-1}\left(\tilde{I}_{\mu, 1} \xi \cup \tilde{I}_{\mu, 2} \xi\right) \\
& \cup \bigcup_{\mu=1}^{m-1} \tilde{I}_{\mu, 1} \xi^{2} \cup \bigcup_{\mu=0}^{m-1} \tilde{I}_{\mu, 2} \xi^{2}
\end{aligned}
$$

2. Let $\varphi_{1}\left(\beta_{1}, \beta_{2}\right)=\left(n_{E}\left(\beta_{1}\right), \beta_{1}{ }^{\sigma} \beta_{2}\right)$ for $\left(\beta_{1}, \beta_{2}\right) \in \varpi_{E}^{\mu} \mathcal{O}_{E}^{\times} \times P_{E}^{\mu}$ and $\varphi_{2}\left(\beta_{1}, \beta_{2}\right)=$ $\left(\beta_{2} \sigma^{2} \beta_{1}, n_{E}\left(\beta_{2}\right)\right)$ for $\left(\beta_{1}, \beta_{2}\right) \in P_{E}^{\mu+1} \times \varpi_{E}^{\mu} \mathcal{O}_{E}^{\times}$. Then $\varphi_{i}$ induces a bijection from $I_{\mu, i}$ to $J_{\mu, i}$ for $i=1,2$.

Proof. Since $H\left(1+\beta_{1} \xi+\beta_{2} \xi^{2}\right) \alpha=H\left(1+\beta_{1}{ }^{\sigma} \alpha \alpha^{-1} \xi+\beta_{2} \sigma^{2} \alpha \alpha^{-1} \xi^{2}\right)$ for any $\alpha \in E^{\times}$ and any element of $B / E^{\times}$has a representative of the form $\left(1+\beta_{i 1} \xi+\beta_{i 2} \xi^{2}\right) \xi^{i}$ $(0 \leq i \leq 2)$, where $\beta_{01}, \beta_{02}, \beta_{22} \in \mathcal{O}_{E}$ and $\beta_{11}, \beta_{12}, \beta_{21} \in P_{E}$, the first part of the lemma follows from Lemma 2.2. To prove the second part of the lemma, it suffices to see that $\varphi_{1}$ induces a bijection from $I_{0,1}$ to $J_{0,1}$. Let $\beta_{1}, \gamma_{1} \in \mathcal{O}_{E}^{\times}$and $\beta_{2}, \gamma_{2} \in \mathcal{O}_{E}$. If $\left(\gamma_{1}, \gamma_{2}\right)$ belongs to the double coset $\left(\left(1+P_{E}^{m}\right) \times P_{E}^{m}\right)\left(\beta_{1}, \beta_{2}\right) M$, 
then there exist $\alpha \in \mathcal{O}_{E}^{\times}$and $y_{1}, y_{2} \in P_{E}^{m}$ such that

$$
\begin{aligned}
& \gamma_{1}={ }^{\sigma} \alpha \alpha^{-1} \beta_{1}\left(1+y_{1}\right), \\
& \gamma_{2}={ }^{\sigma^{2}} \alpha \alpha^{-1}\left(\beta_{2}+y_{2}\right) .
\end{aligned}
$$

This implies

$$
\begin{aligned}
n_{E}\left(\beta_{1}\right) & \equiv n_{E}\left(\gamma_{1}\right) \quad \bmod 1+P_{E}^{m} \quad \text { (multiplicative equivalence), } \\
\gamma_{1}{ }^{\sigma} \gamma_{2} & \equiv \beta_{1}{ }^{\sigma} \beta_{2} \quad \bmod P_{E}^{m} .
\end{aligned}
$$

Therefore $\varphi_{1}$ induces a well-defined map from $I_{0,1}$ to $J_{0,1}$. The induced map's bijectivity follows from the bijectivity of the map

$$
\mathcal{O}_{E}^{(1)} \backslash \mathcal{O}_{E}^{\times} /\left(1+P_{E}^{j}\right) \stackrel{n_{E}}{\longrightarrow} n_{E}\left(\mathcal{O}_{E}^{\times}\right) /\left(1+P_{F}^{[(j+2) / 3]}\right) .
$$

Next we consider the term $a^{-1} H a \cap E^{\times}$.

Lemma 2.4. Let $a \in \tilde{I}_{\mu, j}(j=1$ or 2 and $0 \leq \mu<m)$. Then $a^{-1} H a \cap E^{\times}=$ $F^{\times}\left(1+P_{E}^{m-\mu}\right)$.

Proof. Let $a=1+\beta_{1} \xi+\beta_{2} \xi^{2} \in \tilde{I}_{\mu, j}$ and let $\alpha \in a^{-1} H a \cap E^{\times}$. Let us begin by showing that $\alpha \in F^{\times}\left(1+P_{E}^{m-\mu}\right)$. First, since $F^{\times} \subset E^{\times}$, we may assume that $0 \leq v_{E}(\alpha) \leq 2$. Since $a \alpha=\gamma a$, where $\gamma=\gamma_{0}+\gamma_{1} \xi+\gamma_{2} \xi^{2} \in H$, we have $\gamma_{i} \in E^{\times}$ $(i=0,1,2)$ such that $\gamma_{0}^{-1} \gamma_{i} \in P_{E}^{m}(i=1,2)$ and $v_{E}\left(\gamma_{0}\right)=v_{E}(\alpha)$. From this we see that

$$
\begin{aligned}
\gamma_{0} & =\alpha-\left(\gamma_{1}{ }^{\sigma} \beta_{2}+\gamma_{2}{ }^{\sigma^{2}} \beta_{1}\right), \\
\left({ }^{\sigma} \alpha-\gamma_{0}\right) \beta_{1} & =\gamma_{1}+\gamma_{2}{ }^{\sigma^{2}} \beta_{2}, \\
\left({ }^{\sigma^{2}} \alpha-\gamma_{0}\right) \beta_{2} & =\gamma_{2}+\gamma_{1}{ }^{\sigma} \beta_{1} .
\end{aligned}
$$

Replacing $\gamma_{0}$ by $\alpha-\left(\gamma_{1} \sigma_{\beta_{2}}+\gamma_{2} \sigma^{2} \beta_{1}\right)$, we obtain the relations

$$
\left({ }^{\sigma^{i}} \alpha-\alpha\right) \beta_{i} \in P_{E}^{m+v_{E}(\alpha)} \quad(i=1,2) \text {. }
$$

If $v_{E}(\alpha)=1$ or 2 , then $v_{E}\left({ }^{i} \alpha-\alpha\right)=v_{E}(\alpha)$, which implies that $v_{E}\left(\beta_{i}\right) \geq m$. From this we have a contradiction to the hypothesis that $\mu<m$; so we may assume that $v_{E}(\alpha)=0$. In this case, we see that $v_{E}\left({ }^{i} \alpha-\alpha\right) \geq m-\mu$, and we therefore conclude that $\alpha \in F^{\times}\left(1+P_{E}^{m-\mu}\right)$. Now let us prove the inclusion $F^{\times}\left(1+P_{E}^{m-\mu}\right) \subset a^{-1} H a \cap E^{\times}$. It is enough to take $\alpha \in\left(1+P_{E}^{m-\mu}\right)$ and to show that $a \alpha=\gamma a$ with $\gamma \in H$ as before. If $\mu=0$, then $\left(1+P_{E}^{m}\right) \subset H$ and $a \in K$; since $K$ normalizes $H$, this case is clear. We need consider only $0<\mu<m$. For the proof we intend to reverse the above reasoning. Certainly, we have $v_{E}(\alpha)=0$ and, moreover, $v_{E}\left({ }^{\sigma^{i}} \alpha-\alpha\right) \geq m-\mu$. From this we see that

$$
\left({ }^{\sigma^{i}} \alpha-\alpha\right) \beta_{i} \in P_{E}^{m} \quad(i=1,2) \text {. }
$$

Replacing $\gamma_{0}$ by $\alpha-\left(\gamma_{1}{ }^{\sigma} \beta_{2}+\gamma_{2} \sigma^{2} \beta_{1}\right)$ in the last two equations of (2.11) produces the system of equations

$$
\begin{aligned}
\left({ }^{\sigma} \alpha-\alpha\right) \beta_{1} & =\left(1-\beta_{1}{ }^{\sigma} \beta_{2}\right) \gamma_{1}+\left({ }^{\sigma^{2}} \beta_{2}-{ }^{\sigma^{2}} \beta_{1} \beta_{1}\right) \gamma_{2} \\
\left({ }^{\sigma^{2}} \alpha-\alpha\right) \beta_{2} & =\left({ }^{\sigma} \beta_{1}-{ }^{\sigma} \beta_{2} \beta_{2}\right) \gamma_{1}+\left(1-{ }^{\sigma^{2}} \beta_{1} \beta_{2}\right) \gamma_{2} .
\end{aligned}
$$


Since $\mu>0$, it follows that the determinant of the coefficient matrix of this system of two equations in the variables $\gamma_{1}, \gamma_{2}$ belongs to $\mathcal{O}_{E}^{\times}$. From (2.12) it follows that $\gamma_{1}, \gamma_{2} \in P_{E}^{m}$; obviously, $\gamma_{0} \in \mathcal{O}_{E}^{\times}$, given by the first equation of 2.11), satisfies $\gamma_{0}^{-1} \gamma_{i} \in P_{E}^{m}$.

Our next task is to compute ${ }^{a} \rho$ for $a \in H \backslash B / E^{\times}$. The above lemma tells us that ${ }^{a} \rho \in\left(F^{\times}\left(1+P_{E}^{m-\mu}\right)\right)^{\wedge}$ if $a \in \tilde{I}_{\mu, i}$. If $a^{\prime}=a \xi^{j}$, then $a^{\prime-1} H a^{\prime} \cap E^{\times}=a^{-1} H a \cap E^{\times}$ and ${ }^{a^{\prime}} \rho={ }^{a} \rho \circ \sigma^{j}$. Therefore it suffices to consider ${ }^{a} \rho$ for $a \in \tilde{I}_{\mu, i}$. We need the following explicit form of $\left(1+\alpha_{1} \xi+\alpha_{2} \xi^{2}\right)^{-1}$.

Lemma 2.5. Let $a=1+\alpha_{1} \xi+\alpha_{2} \xi^{2}$ for $\alpha_{1}, \alpha_{2} \in E$. Then

$$
a^{-1}=\frac{1}{\operatorname{det}(a)}\left(\left(1-{ }^{\sigma} \alpha_{1}{ }^{\sigma^{2}} \alpha_{2}\right)+\left(\alpha_{2}{ }^{\sigma^{2}} \alpha_{2}-\alpha_{1}\right) \xi+\left(\alpha_{1}{ }^{\sigma} \alpha_{1}-\alpha_{2}\right) \xi^{2}\right),
$$

where

$$
\operatorname{det}(a)=1+n_{E}\left(\alpha_{1}\right)+n_{E}\left(\alpha_{2}\right)-\operatorname{tr}_{E}\left(\alpha_{1}^{\sigma} \alpha_{2}\right) .
$$

Proof. Put $\left(\beta_{0}+\beta_{1} \xi+\beta_{2} \xi^{2}\right)\left(1+\alpha_{1} \xi+\alpha_{2} \xi^{2}\right)=1$ for $\beta_{i} \in E$. Since $\xi x \xi^{-1}={ }^{\sigma} x$ for $x \in E$, we have

$$
\left(\begin{array}{ccc}
1 & \sigma_{\alpha_{2}} & \sigma^{2} \alpha_{1} \\
\alpha_{1} & 1 & \sigma^{2} \alpha_{2} \\
\alpha_{2} & \sigma_{\alpha_{1}} & 1
\end{array}\right)\left(\begin{array}{c}
\beta_{0} \\
\beta_{1} \\
\beta_{2}
\end{array}\right)=\left(\begin{array}{l}
1 \\
0 \\
0
\end{array}\right) .
$$

Thus the lemma follows from Cramer's rule.

Lemma 2.6. Let $c \in F^{\times}, y \in P_{E}^{m-\mu}, a=1+\beta_{1} \xi+\beta_{2} \xi^{2} \in \tilde{I}_{\mu, i}$ and $\gamma=\gamma_{\theta} \in E$ as in (1.1). Then

$$
\begin{aligned}
{ }^{a} \rho \rho^{-1}(c(1+y)) & =\psi_{E}\left(R_{\mu, 1}\left(\varphi_{2}\left(\beta_{1}, \beta_{2}\right)\right)\left(\sigma^{2} y-y\right)\right) \\
& =\psi_{E}\left(R_{\mu, 2}\left(\varphi_{1}\left(\beta_{1}, \beta_{2}\right)\right)\left({ }^{\sigma} y-y\right)\right),
\end{aligned}
$$

where

$$
\begin{aligned}
& R_{\mu, 1}(u, v)=\frac{\left(\sigma^{2} \gamma-\gamma\right) u+\gamma v-\sigma^{2} \gamma v^{-1} n_{E}(u)}{1+v+v^{-1} n_{E}(u)-\operatorname{tr}_{E}(u)}, \\
& R_{\mu, 2}(v, u)=\frac{\left(\sigma^{\sigma} \gamma-\gamma\right) u+\gamma v-{ }^{\sigma} \gamma v^{-1} n_{E}(u)}{1+v+v^{-1} n_{E}(u)-\operatorname{tr}_{E}(u)}
\end{aligned}
$$

and $\varphi_{i}$ is as in Lemma2.3 (2) for $i=1,2$.

Proof. Putting $g=1+y$, we have

$$
\begin{aligned}
g^{-1} a g a^{-1} & =1+\left(g^{-1}(a-1) g-(a-1)\right) a^{-1} \\
& =1+\left(g^{-1} \beta_{1}{ }^{\sigma} g \xi+g^{-1} \beta_{2} \sigma^{2} g \xi^{2}-\left(\beta_{1} \xi+\beta_{2} \xi^{2}\right)\right) a^{-1} \\
& =1+\left(\left({ }^{\sigma} g g^{-1}-1\right) \beta_{1} \xi+\left({ }^{\sigma^{2}} g g^{-1}-1\right) \beta_{2} \xi^{2}\right) a^{-1} .
\end{aligned}
$$

Using Lemma 2.5 we see that $\operatorname{det}(a)\left(g^{-1} a g a^{-1}-1\right)$ equals

$$
\begin{aligned}
& \left(\left({ }^{\sigma} g g^{-1}-1\right) \beta_{1} \xi+\left({ }^{\sigma^{2}} g g^{-1}-1\right) \beta_{2} \xi^{2}\right) \\
& \times\left(\left(1-{ }^{\sigma} \beta_{1}{ }^{\sigma^{2}} \beta_{2}\right)+\left({\beta_{2}}^{\sigma^{2}} \beta_{2}-\beta_{1}\right) \xi+\left(\beta_{1}{ }^{\sigma} \beta_{1}-\beta_{2}\right) \xi^{2}\right) .
\end{aligned}
$$


Since ${ }^{\sigma^{j}} g g^{-1}-1 \equiv \sigma^{j} y-y \bmod P_{E}^{2(m-\mu)}, \beta_{i} \in P_{E}^{\mu},\left.\rho\right|_{K^{m}}=\psi_{\gamma}, \operatorname{tr}_{E}\left(x \xi^{i}\right)=0$ for $i=1,2$ and $x \in E$, we get

$$
\begin{aligned}
& { }^{a} \rho \rho^{-1}(1+y) \\
& \quad=\psi_{\gamma}\left(\frac{1}{\operatorname{det}(a)}\left(\beta_{1}\left({ }^{\sigma} \beta_{1} \sigma^{2} \beta_{1}-{ }^{\sigma} \beta_{2}\right)\left({ }^{\sigma} y-y\right)+\beta_{2}\left({ }^{\sigma^{2}} \beta_{2}{ }^{\sigma^{4}} \beta_{2}-{ }^{\sigma^{2}} \beta_{1}\right)\left(\sigma^{\sigma^{2}} y-y\right)\right)\right) \\
& \quad=\psi_{\gamma}\left(\frac{1}{\operatorname{det}(a)}\left(\left(n_{E}\left(\beta_{1}\right)-\beta_{1}{ }^{\sigma} \beta_{2}\right)\left({ }^{\sigma} y-y\right)+\left(n_{E}\left(\beta_{2}\right)-\beta_{2}{ }^{2} \beta_{1}\right)\left({ }^{\sigma^{2}} y-y\right)\right)\right) .
\end{aligned}
$$

Since $\operatorname{tr}_{E} u^{\sigma^{j}} v=\operatorname{tr}_{E}{ }^{\sigma^{-j}} u v$ for any $u, v \in E$, we have

$$
\operatorname{tr}_{E}\left(\gamma\left(n_{E}\left(\beta_{1}\right)-\beta_{1}{ }^{\sigma} \beta_{2}\right)\left({ }^{\sigma} y-y\right)\right)=\operatorname{tr}_{E}\left({ }^{\sigma^{2}} \gamma\left(n_{E}\left(\beta_{1}\right)-{ }^{\sigma^{2}} \beta_{1} \beta_{2}\right)\left(y-{ }^{\sigma^{2}} y\right)\right) .
$$

Therefore we get

$$
{ }^{a} \rho \rho^{-1}(1+y)=\psi_{E}\left(\frac{\left(\sigma^{2} \gamma-\gamma\right)^{\sigma^{2}} \beta_{1} \beta_{2}+\gamma n_{E}\left(\beta_{2}\right)-\sigma^{2} \gamma n_{E}\left(\beta_{1}\right)}{\operatorname{det}(a)}\left(\sigma^{2} y-y\right)\right) .
$$

From $\operatorname{det}(a)=1+n_{E}\left(\beta_{1}\right)+n_{E}\left(\beta_{2}\right)-\operatorname{tr}_{E}\left(\beta_{1} \sigma \beta_{2}\right)$, we obtain (2.14). The equation (2.13) can be shown in the same way.

The next lemma is crucial for the character formula of $\kappa=\kappa_{\theta}$. We recall that $m=[(n+2) / 2]$.

Lemma 2.7. Fix $\alpha \in \varpi_{F}^{\mu} n_{E}\left(\mathcal{O}_{E}^{\times}\right)$and put $\tilde{R}_{\mu, i}(x)=R_{\mu, i}(x, \alpha)$ for $x \in P_{E}^{2 \mu+i-1}$. For $\mu=0$, we assume $1+\alpha+\alpha^{-1} x^{3}-3 x \notin P_{E}$.

1. If $\mu \geq 1$, then $\tilde{R}_{\mu, i}$ induces a bijection from $P_{E}^{2 \mu+i-1} / P_{E}^{m+\mu}$ to $P_{E}^{2 \mu+i-1-n} /$ $P_{E}^{m+\mu-n}$.

2. $\tilde{R}_{0,2}$ induces a bijection from $P_{E} / P_{E}^{m}$ to

$$
\left\{x \in P_{E}^{-n} / P_{E}^{m-n} \mid x \equiv \frac{\gamma \alpha}{1+\alpha} \bmod P_{E}^{1-n}\right\} .
$$

3. For any $x_{0} \in \mathcal{O}_{E}$ such that $1+\alpha+\alpha^{-1} x_{0}^{3}-3 x_{0} \notin P_{E}, \tilde{R}_{0,1}$ induces a bijection from $\left\{x \in \mathcal{O}_{E} / P_{E}^{m} \mid x \equiv x_{0} \bmod P_{E}\right\}$ to

$$
\left\{x \in P_{E}^{-n} / P_{E}^{m-n} \mid x \equiv \frac{\left(\sigma^{2} \gamma-\gamma\right) x_{0}+\gamma \alpha-{ }^{\sigma^{2}} \gamma \alpha^{-1} x_{0}^{3}}{1+\alpha+\alpha^{-1} x_{0}^{3}-3 x_{0}} \quad \bmod P_{E}^{1-n}\right\} .
$$

Proof. First we assume $\mu>0$. Let $x \in P_{E}^{2 \mu+i-1}$. Then

$$
\tilde{R}_{\mu, i}(x) \equiv\left({ }^{\sigma^{-i}} \gamma-\gamma\right) x \bmod P_{E}^{v_{E}(x)-n+1} .
$$

We remark that $v_{E}\left({ }^{\sigma^{-i}} \gamma-\gamma\right)=-n$, since $n \not \equiv 0 \bmod 3$. It follows from (2.15) that

$$
\tilde{R}_{\mu, i}\left(x_{1}\right) \equiv \tilde{R}_{\mu, i}\left(x_{2}\right)+\left(\sigma^{-i} \gamma-\gamma\right)\left(x_{1}-x_{2}\right) \quad \bmod P_{E}^{v_{E}\left(x_{1}\right)+v_{E}\left(x_{2}-x_{1}\right)+i-n}
$$

if $2 \mu+i-1 \leq v_{E}\left(x_{1}\right) \leq v_{E}\left(x_{2}\right)$. This implies $x_{1}-x_{2} \in P_{E}^{m}$ if $\tilde{R}_{\mu, i}\left(x_{1}\right)-\tilde{R}_{\mu, i}\left(x_{2}\right)$ $\in P_{E}^{m-n}$. Thus the induced map from $P_{E}^{2 \mu+i-1} / P_{E}^{m+\mu}$ to $P_{E}^{2 \mu+i-1-n} / P_{E}^{m+\mu-n}$ is injective. It becomes automatically bijective.

Next we consider the case $\mu=0$. For $x \in P_{E}$,

$$
\tilde{R}_{0,2}(x) \equiv \frac{\gamma \alpha+\left({ }^{\sigma} \gamma-\gamma\right) x}{1+\alpha} \bmod P_{E}^{v_{E}(x)+1-n} .
$$

This implies the bijectivity of the induced map from $\tilde{R}_{0,2}$ by the same argument as above. 
For $x_{0} \in \mathcal{O}_{E}^{\times}$and $x_{1} \in P_{E}$,

$$
\begin{aligned}
\tilde{R}_{0,1}\left(x_{0}+x_{1}\right) & =\frac{\left(\sigma^{2} \gamma-\gamma\right)\left(x_{0}+x_{1}\right)+\gamma \alpha-\sigma^{2} \gamma \alpha^{-1} n_{E}\left(x_{0}\right) n_{E}\left(1+x_{0}^{-1} x_{1}\right)}{1+\alpha+\alpha^{-1} n_{E}\left(x_{0}\right) n_{E}\left(1+x_{0}^{-1} x_{1}\right)-\operatorname{tr}_{E}\left(x_{0}+x_{1}\right)} \\
& \equiv \frac{\left(\sigma^{2} \gamma-\gamma\right) x_{0}+\gamma \alpha-{ }^{2} \gamma \alpha^{-1} n_{E}\left(x_{0}\right)+\left(\sigma^{2} \gamma-\gamma\right) x_{1}}{1+\alpha+\alpha^{-1} n_{E}\left(x_{0}\right)-\operatorname{tr}_{E}\left(x_{0}\right)} \bmod P_{E}^{2-n} \\
& \equiv \tilde{R}_{0,1}\left(x_{0}\right)+\frac{\left(\sigma^{2} \gamma-\gamma\right) x_{1}}{1+\alpha+\alpha^{-1} n_{E}\left(x_{0}\right)-\operatorname{tr}_{E}\left(x_{0}\right)} \quad \bmod P_{E}^{2-n},
\end{aligned}
$$

since $n_{E}\left(1+x_{0}^{-1} x_{1}\right)$ and $\operatorname{tr}_{E}\left(x_{1}\right) \in P_{F} \subset P_{E}^{3}$. Thus the last assertion also follows in the same way as the proof for the case $\mu>0$.

Now we can describe the image of the map $a \mapsto{ }^{a} \rho \rho^{-1}$ for $a \in \tilde{I}_{\mu, i}$.

Lemma 2.8. Let $U_{i}=F^{\times}\left(1+P_{E}^{i}\right)$ for $i>0$ and $U_{0}=F^{\times} \mathcal{O}_{E}^{\times}$. For $\mu \geq 0$ and $i \in\{1,2\}$, we put $C(\mu, i)=q^{[(m-\mu-1) / 3]+[(m+\mu-n+2) / 3]-[(2 \mu+i+1-n) / 3]}$.

1. For $\mu>0$,

$$
\bigoplus_{a \in \tilde{I}_{\mu, i}}{ }^{a} \rho \rho^{-1}=\frac{q-1}{3} C(\mu, i)\left|P_{E}^{\mu-m+1} \cap F / P_{E}^{m+\mu-n} \cap F\right| \underset{\chi \in(U(m-\mu) / U(n+2-2 \mu-i))^{\wedge}}{\bigoplus^{\wedge}} \chi .
$$

2. For $\alpha \in n_{E}\left(\mathcal{O}_{E}^{\times}\right)$, put

$$
\Lambda(\alpha)=\left\{\chi \in\left(U_{m} / U_{n+1}\right)^{\wedge} \mid \chi(1+y)=\psi_{E}\left(\frac{\gamma \alpha}{1+\alpha}\left(\sigma^{2} y-y\right)\right) \text { for } y \in P_{E}^{n}\right\} .
$$

Then

$$
\bigoplus_{a \in \tilde{I}_{0,2}}{ }^{a} \rho \rho^{-1}=C(0,2) \bigoplus_{\alpha \in n_{E}\left(\mathcal{O}_{E}^{\times}\right) / 1+P_{F}} \bigoplus_{\chi \in \Lambda(\alpha)} \chi
$$

3. For $\alpha \in n_{E}\left(\mathcal{O}_{E}^{\times}\right)$and $x_{0} \in \mathcal{O}_{E}$ satisfying $1+\alpha+\alpha^{-1} x_{0}^{3}-3 x_{0} \notin P_{E}$, let $\Omega\left(\alpha, x_{0}\right)$ be a subset of $\left(U_{m} / U_{n+1}\right)^{\wedge}$ consisting of characters $\chi$ such that

$$
\chi(1+y)=\psi_{E}\left(\frac{\left(\sigma^{2} \gamma-\gamma\right) x_{0}+\gamma \alpha-\sigma^{2} \gamma \alpha^{-1} x_{0}^{3}}{1+\alpha+\alpha^{-1} x_{0}^{3}}\left({ }^{\sigma} y-y\right)\right) \quad \text { for } y \in P_{E}^{n} .
$$

Then

$$
\bigoplus_{a \in \tilde{I}_{0,1}}{ }^{a} \rho \rho^{-1}=C(0,2) \bigoplus_{\alpha \in n_{E}\left(\mathcal{O}_{E}^{\times}\right) / 1+P_{F}} \bigoplus_{x_{0} \in \mathcal{O}_{E} / P_{E}} \bigoplus_{\chi \in \Omega\left(\alpha, x_{0}\right)} \chi .
$$

Proof. By Lemmas 2.7 and 2.8, it suffices to calculate the multiplicity of the character $\chi$ in the right-hand side. The map

$$
\begin{aligned}
& P_{E}^{2 \mu-i-1-n} / P_{E}^{m+\mu-n} \rightarrow\left(U_{m-\mu} / U_{n+1-2 \mu+1-i}\right)^{\wedge}, \\
& a \mapsto\left(c(1+y) \mapsto \psi_{E}\left(a\left({ }^{\sigma} y-y\right)\right)\right),
\end{aligned}
$$

is surjective and every fiber of the map has

$$
\left|P_{E}^{2 \mu-i-1-n} \cap F / P_{E}^{m+\mu-n} \cap F\right|\left|P_{E}^{\mu-m+1} \cap F / P_{E}^{m+\mu-n} \cap F\right|
$$

elements. Since $P_{E}^{j} \cap F=P_{F}^{[(j+2) / 3]},\left|n_{E}\left(\mathcal{O}_{E}^{\times}\right) / 1+P_{F}^{[(m-\mu+2) / 3]}\right|=\frac{q-1}{3} q^{[(m-\mu-1) / 3]}$,

$$
m+\mu-n=\left\{\begin{array}{lll}
\mu-m+1 & \text { if } & n=2 m-1, \\
\mu-m+2 & \text { if } & n=2 m-2,
\end{array}\right.
$$


and $m \not \equiv 1 \bmod 3$ when $n=2 m-2$, we get this lemma from Lemma [2.6] and Lemma 2.7

Now we can give the character formula of $\kappa=\kappa_{\theta}$ on $E^{\times}$.

Proposition 2.9. Put $C=q^{[(m-\mu-1) / 3]+[(m+\mu-n+2) / 3]-[(2 \mu+i+1-n) / 3]}$ for $\mu \geq 0$ and $i \in\{1,2\}$.

(1) (Decomposition of $\kappa$ as $E^{\times}$-module) The restriction of $\kappa$ to $E^{\times}$is decomposed as follows:

$$
\begin{aligned}
& \left(\bigoplus_{i=0}^{2} \theta \circ \sigma^{i}\right) \otimes\left(\{1\}+\sum_{j=1}^{n-1} \frac{q-1}{3} q^{c_{j}} X_{j}\right) \\
& \oplus C\left(\theta \otimes \bigoplus_{\alpha \in n_{E}\left(\mathcal{O}_{E}^{\times}\right) / 1+P_{F}} \bigoplus_{\chi \in \tilde{\Lambda}(\alpha)} \chi\right. \\
& \oplus\left(\theta \circ \sigma^{2}\right) \otimes \bigoplus_{\alpha \in n_{E}\left(\mathcal{O}_{E}^{\times}\right) / 1+P_{F}} \bigoplus_{\chi \in \tilde{\Lambda}(\alpha)} \chi \\
& \left.\oplus \theta \otimes \bigoplus_{\alpha \in n_{E}} \bigoplus_{\left(\mathcal{O}_{E}^{\times}\right) / 1+P_{F}} \bigoplus_{x_{0} \in \mathcal{O}_{E} / P_{E}} \chi\right),
\end{aligned}
$$

where $X_{j}=\bigoplus_{\chi \in E^{\times} / U_{j}} \chi, c_{j}=2\left[\frac{j+2}{6}\right]$,

$$
\tilde{\Lambda}(\alpha)=\left\{\chi \in\left(E^{\times} / U_{n+1}\right)^{\wedge} \mid \chi(1+y)=\psi_{E}\left(\frac{\gamma \alpha}{1+\alpha}\left(\sigma^{2} y-y\right)\right) \text { for } y \in P_{E}^{n}\right\}
$$

and $\Omega\left(\alpha, x_{0}\right)$ is a subset of $\left(E / U_{n+1}\right)^{\wedge}$ consisting of characters $\chi$ such that

$$
\chi(1+y)=\psi_{E}\left(\frac{\left(\sigma^{2} \gamma-\gamma\right) x_{0}+\gamma \alpha-{ }^{\sigma^{2}} \gamma \alpha^{-1} x_{0}^{3}}{1+\alpha+\alpha^{-1} x_{0}^{3}}\left({ }^{\sigma} y-y\right)\right) \text { for } y \in P_{E}^{n}
$$

(2) (Character formula of $\kappa$ on $E^{\times}$) The character $\chi_{\kappa}$ of $\kappa$ is given by

\begin{tabular}{|c|c|}
\hline$x$ & $\chi_{\kappa}(x)$ \\
\hline$x \in E^{\times}-U_{0}$ & $\sum_{i=0}^{2} \theta\left({ }^{\sigma^{i}} x\right)$ \\
\hline$x \in U_{j}^{*} \quad(1 \leq j \leq n-1)$ & $q^{j} \sum_{i=0}^{2} \theta\left({ }^{i} x\right)$ \\
\hline$c(1+y) \in U_{n}^{*}\left(c \in F^{\times}, y \in \varpi_{E}^{n} \mathcal{O}_{E}^{\times}\right)$ & $q^{n-1} \theta(c) \mathrm{Kl}(\gamma y)$ \\
\hline$x \in U_{n+1}$ & $q^{n-1}(q-1)^{2} \theta(x)$ \\
\hline
\end{tabular}

where the Kloosterman sum $\mathrm{Kl}(a)$ is defined by

$$
\mathrm{Kl}(a)=\sum_{\substack{\left(y_{0}, y_{1}, y_{2}\right) \in k_{F}^{3} \\ y_{0} y_{1} y_{2}=a}} \psi\left(\gamma \varpi_{E}^{n}\left(y_{0}+y_{1}+y_{2}\right)\right)
$$

(Since $\gamma \varpi_{E}^{n} \in \mathcal{O}_{E}$ and $k_{E}=k_{F}$, we regard $\gamma \varpi_{E}^{n} \bmod P_{E}$ as an element of $k_{F}$. ) 
Proof. If $n=2 m-1$, then it follows from Lemma 2.8 that

$$
\begin{aligned}
& \bigoplus_{a \in \tilde{I}_{\mu, i}} \operatorname{Ind}_{U_{m-\mu}}^{E^{\times}}{ }^{a} \rho \rho^{-1}
\end{aligned}
$$

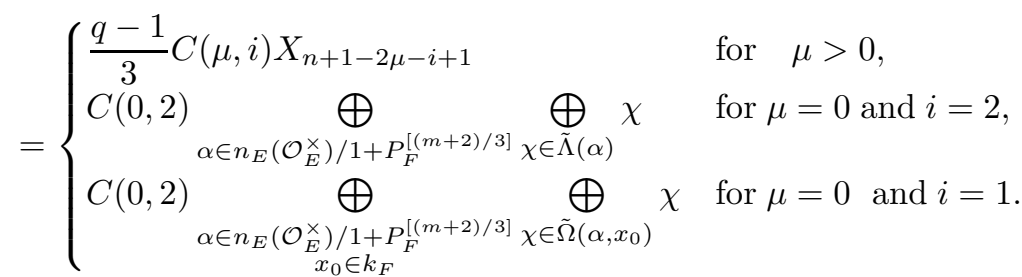

By virtue of (2.7) and (2.3), we can obtain the decomposition of $\pi$ as an $E^{\times}$-module. When $n=2 m-2$, we get by Lemma 2.8 that

$$
\begin{aligned}
& \bigoplus_{a \in \tilde{I}_{\mu, i}} \operatorname{Ind}_{U_{m-\mu}}^{E^{\times}}{ }^{a} \rho \rho^{-1}
\end{aligned}
$$

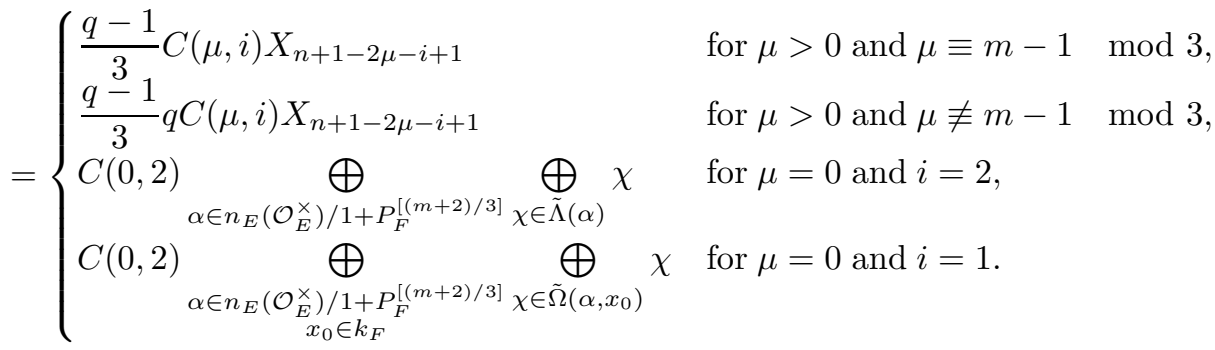

By (2.8) and (2.3), we get the first part of the theorem. Except on $U_{n}^{*}$, we can easily obtain the character formula of $\kappa$ from the decomposition of $\kappa$ as an $E^{\times}$-module, Lemma 2.8 and the fact that $\left|E^{\times} / U_{j}\right|=3 q^{[2 j / 3]}$. For $x \in U_{n}^{*}$, we can prove it by the decomposition of $\kappa$ and Lemma [2.6] But the calculation of the character on $K^{n}$ can be obtained directly from the definition of the induced representation. We state it, including the case $x \notin E$.

Lemma 2.10. Let $x=1+\varpi_{E}^{n} x_{0}$ for $x_{0}=\operatorname{diag}\left(k_{1}, k_{2}, k_{3}\right)\left(k_{i} \in \mathcal{O}_{F}^{\times}\right)$and the Kloosterman sum $\mathrm{Kl}(a)$ as in (2.17). Then

$$
\chi_{\kappa}(x)=q^{n-1} \mathrm{Kl}\left(k_{1} k_{2} k_{3}\right) .
$$

Proof. By the definition of $\kappa$ and Frobenius's formula, we have

$$
\chi_{\kappa}\left(1+\varpi_{E}^{n} \operatorname{diag}\left(k_{1}, k_{2}, k_{3}\right)\right)=q^{n-1} \sum_{a \in E^{\times} K^{1} \backslash B} \psi\left(\operatorname{Tr} \gamma a \varpi_{E}^{n} \operatorname{diag}\left(k_{1}, k_{2}, k_{3}\right) a^{-1}\right) .
$$

It follows from (2.3) and (2.5) that the set $\left\{\operatorname{diag}(1, y, z) \mid y, z \in k_{F}^{\times}\right\}$becomes a complete set of representatives of $E^{\times} K^{1} \backslash B$. Since $\varpi_{E} \operatorname{diag}(1, y, z) \varpi_{E}^{-1}=\operatorname{diag}(y, z, 1)$, we have

$$
\begin{aligned}
& \operatorname{Tr} \gamma \operatorname{diag}(1, y, z) \varpi_{E}^{n} \operatorname{diag}\left(k_{1}, k_{2}, k_{3}\right) \operatorname{diag}(1, y, z)^{-1} \bmod P_{F} \\
& =\left\{\begin{array}{llll}
\gamma \varpi_{E}^{n}\left(z k_{1}+y^{-1} k_{2}+y z^{-1} k_{3}\right) & \bmod P_{F} & \text { if } n \equiv 1 \bmod 3, \\
\gamma \varpi_{E}^{n}\left(y k_{1}+z y^{-1} k_{2}+z^{-1} k_{3}\right) & \bmod P_{F} & \text { if } n \equiv 2 & \bmod 3 .
\end{array}\right.
\end{aligned}
$$

Hence our lemma. 
By applying the above lemma for $\operatorname{diag}\left(k_{1}, k_{2}, k_{3}\right)=\operatorname{diag}\left(x_{0}, x_{0}, x_{0}\right)$, we get the character formula of $\kappa$ on $U_{n}^{*}$.

The character outside of the conjugacy class of $E^{\times}$can be obtained easily.

Lemma 2.11. Let $x$ be a regular elliptic element of $B$. If $x$ satisfies the conditions that $F(x) \not E$ and $x$ is not conjugate to an element of $F^{\times} K^{n}$, then $\chi_{\kappa}(x)=0$.

Proof. See Lemma 3.3 in $[15]$.

We remark that if $F(x) \mid F$ is unramified, then $F(x) \cap K^{n}=F(x) \cap K^{n+1}$ since $n \not \equiv 0 \bmod 3$. Let $E^{\prime} \mid F$ be a ramified extension in $\mathrm{M}_{3}(F)$. Then either $E^{\prime} \sim E$ or $E^{\prime} \sim F(x)$, where $x=\varpi_{E} \operatorname{diag}(b, 1,1)$ with $b \in \mathcal{O}_{F}^{\times}$not a cube $\bmod P_{F}$. The element $x$ is obviously a prime element of $\mathcal{O}_{F(x)}$. If $g \in F(x)$ and $v_{F(x)}(g-1)=n$, then $g=1+x^{n} y+z$ for some $y \in k_{F}^{\times}$and $z \in P_{F(x)}^{n+1}$.

Proposition 2.12. Let $x$ be a regular elliptic element of $B$ such that $F(x) \neq E$.

1. If $F(x) \mid F$ is unramified, $\chi_{\kappa}$ is given by

\begin{tabular}{|c|c|}
\hline$x$ & $\chi_{\kappa}(x)$ \\
\hline$\left.x \notin F^{\times}\left(K^{n+1} \cap F(x)\right)\right)$ & 0 \\
\hline$x=c y\left(c \in F^{\times}, y \in K^{n+1} \cap F(x)\right)$ & $q^{n-1}(q-1)^{2}$ \\
\hline
\end{tabular}

2. If $F(x) \mid F$ is ramified, then $\chi_{\kappa}$ is given by

\begin{tabular}{|c|c|}
\hline$x$ & $\chi_{\kappa}(x)$ \\
\hline$x \notin F^{\times}\left(1+P_{F(x)}^{n}\right)$ & 0 \\
\hline$c\left(1+\varpi_{F(x)}^{n} \operatorname{diag}\left(k_{1}, k_{2}, k_{3}\right)+z\right)$ & $q^{n-1} \theta(c) \mathrm{Kl}\left(b^{3[(n+4) / 3} k_{1} k_{2} k_{3}\right)$ \\
$\left(c \in F^{\times}, k_{i} \in k_{F}^{\times}, z \in P_{F(x)}^{n+1}\right)$ & \\
\hline$c(1+y)$ for $c \in F^{\times}, y \in P_{F(x)}^{n+1}$ & $q^{n-1}(q-1)^{2} \theta(c)$ \\
\hline
\end{tabular}

Proof. This follows from Lemma 2.11, Lemma 2.10] and the remark above this proposition.

As pointed out in Theorem 1.6, most of the character formula for $\pi=\pi_{\theta}$ (on the regular elliptic set) comes from the formula for $\kappa=\kappa_{\theta}$.

Theorem 2.13. Let $E$ be a cubic ramified extension of $F$, let $\theta$ be a generic quasicharacter of $E^{\times}$such that $f(\theta)=n+1$, and let $\pi_{\theta}$ be the corresponding irreducible supercuspidal representation of $\mathrm{GL}_{3}(F)$ (see Theorem 1.3). Put $U_{0}=F^{\times} \mathcal{O}_{E}^{\times}, U_{j}=$ $F^{\times}\left(1+P_{E}^{j}\right)$ and $U_{j}^{*}=U_{j}-U_{j+1}$ for $j \geq 1$. Let $\xi$ be an element of $\mathrm{GL}_{3}(F)$ such that $\xi^{3}=1$ and ${ }^{\sigma} x=\xi x \xi^{-1}$ for $x \in E$, where $\langle\sigma\rangle=\operatorname{Gal}(E \mid F)$. Let $x$ be a regular elliptic element of $\mathrm{GL}_{3}(F)$.

1. If $F(x) \mid F$ is unramified, then

\begin{tabular}{|c|c|}
\hline$x$ & $\chi_{\pi_{\theta}}(x)$ \\
\hline$x \notin F^{\times}\left(1+P_{F(x)}^{n+1}\right)$ & 0 \\
\hline$c(1+y)\left(c \in F^{\times}, y \in P_{F(x)}^{n+1}\right)$ & $q^{n-1}\left(q^{2}+q+1\right) \theta(c)$ \\
\hline
\end{tabular}


2. If $x \in E$, then

\begin{tabular}{|c|c|}
\hline$x$ & $\chi_{\pi_{\theta}}(x)$ \\
\hline$x \in E^{\times}-U_{0}$ & $\sum_{i=0}^{2} \theta\left(\sigma^{i} x\right)$ \\
\hline$x \in U_{j}^{*} \quad(1 \leq j \leq n-1)$ & $q^{j} \sum_{i=0}^{2} \theta\left(\sigma^{i} x\right)$ \\
\hline$c(1+y) \in U_{n}^{*}\left(c \in F^{\times}, y \in \varpi_{E}^{n} \mathcal{O}_{E}^{\times}\right)$ & $q^{n-1} \theta(c) \operatorname{Kl}(\gamma y)$ \\
\hline$x \in U_{n+1}$ & $q^{n-1}\left(q^{2}+q+1\right) \theta(x)$ \\
\hline
\end{tabular}

where the Kloosterman sum $\mathrm{Kl}(a)$ is defined by (2.17).

3. If $F(x) \mid F$ is ramified and $F(x) \nsucceq E$, let $b$ be as in the remark above this proposition and put $\varpi_{F(x)}=\varpi_{E} \operatorname{diag}(b, 1,1)$. Then

\begin{tabular}{|c|c|}
\hline$x$ & $\chi_{\pi_{\theta}}(x)$ \\
\hline$x \notin F^{\times}\left(1+P_{F(x)}^{n}\right)$ & 0 \\
\hline$c\left(1+\varpi_{F(x)}^{n} \operatorname{diag}\left(k_{1}, k_{2}, k_{3}\right)+z\right)$ & $q^{n-1} \theta(c) \mathrm{Kl}\left(b^{3[(n+4) / 3} k_{1} k_{2} k_{3}\right)$ \\
$\left(c \in F^{\times}, k_{i} \in k_{F}^{\times}, z \in P_{F(x)}^{n+1}\right)$ & \\
\hline$c(1+y)$ for $c \in F^{\times}, y \in P_{F(x)}^{n+1}$ & $q^{n-1}\left(q^{2}+q+1\right) \theta(c)$ \\
\hline
\end{tabular}

Proof. Except on $K^{n+1} \cap F(x)$ the formula of this theorem follows from Theorem 1.8 and the character formula for $\kappa$, given in Propositions 2.9 and2.12 To complete the formula by determining it on $K^{n+1} \cap F(x)$, we use the abstract matching theorem ([16], 8] and [2]) and the fact, which we have already remarked, that in our case the Howe-Moy correspondence respects abstract matching. The representation $\pi_{\theta}^{\prime}$ of $D^{\times}$which corresponds to the generic character $\theta$ has dimension $q^{n-1}\left(q^{2}+q+1\right)$ and is trivial on $1+P_{D}^{n+1}$. Therefore, by Proposition $1.5, \chi_{\pi_{\theta}}(x)=q^{n-1}\left(q^{2}+q+1\right)$ for all regular elliptic $x \in K_{n+1}$.

\section{Character formula for the non-Galois Case}

In this section we treat the case in which $F$ does not contain cube roots of unity, i.e., the case in which $q=p^{f} \equiv 2 \bmod 3$. In this case the extension $E \mid F$ is not Galois; so we cannot use the methods of the previous section. In order to apply the results obtained in the Galois case we use the base change lift of $\left.\kappa_{\theta}\right|_{B^{1}}$. Let $L$ be an unramified quadratic extension of $F$. Then $L=F(\zeta)$, where $\zeta$ is, as before, a primitive cube root of unity. We write $\tau$ for a generator of $\operatorname{Gal}(L \mid F)\left({ }^{\tau} \zeta=\zeta^{2}\right)$ and add the subscript " $L$ " to objects which are extended by $L$, e.g. $E_{L}=E \otimes_{F} L \cong E L$. The field extension $E_{L} \mid L$ is ramified and Galois, $E_{L} \mid E$ is a quadratic unramified extension, and $\langle\tau\rangle \cong \operatorname{Gal}\left(E_{L} \mid E\right) \cong \operatorname{Gal}(L \mid F)$. Thus $E_{L} \mid F$ is an $\mathfrak{S}_{3}$ extension. We embed $E \hookrightarrow E_{L}$ by sending $x \mapsto x \otimes 1$ and, as in the previous section, we identify $\mathrm{M}_{3}(L)=\mathrm{M}_{3}(F) \otimes_{F} L$ with $\operatorname{End}_{L}\left(E_{L}\right)$. Similarly, we set $G_{L}=\mathrm{GL}_{3}(L)$ and use the $L$-basis $\left\{\varpi_{E}^{2}, \varpi_{E}, 1\right\}$ of $E_{L}$ to identify $G_{L}$ with $\operatorname{Aut}_{L}\left(E_{L}\right)$; we note that this basis is also an $\mathcal{O}_{L}$-basis for $\mathcal{O}_{E_{L}}$. Using the lattice flag $\left\{P_{E_{L}}^{i}\right\}_{i \in \mathbb{Z}}$, we define

$$
A_{L}^{i}=\left\{f \in \mathrm{M}_{3}(L) \mid f\left(P_{E_{L}}^{j}\right) \subset P_{E_{L}}^{j+i} \text { for all } j \in \mathbb{Z}\right\} .
$$

Put $K_{L}=\left(A_{L}^{0}\right)^{\times}, B_{L}=E_{L}^{\times} K_{L}$ and $K_{L}^{i}=1+A_{L}^{i}$ for $i \geq 1$. By Kutzko [15], it suffices to calculate the character of $\kappa=\kappa_{\theta}$ instead of $\pi_{\theta}$. In fact, we have only to determine the character of $\left.\kappa\right|_{B^{1}}$. Therefore we have only to treat the base change of $\left.\kappa\right|_{B^{1}}$ to $B_{L}^{1}$, where $B_{L}^{1}=L^{\times} K_{L}$. 
Definition 3.1. Let $\theta$ be a generic character of $E^{\times}$with $f(\theta)=n+1$ and $\theta(1+x)=\psi\left(\operatorname{tr}_{E}(\gamma x)\right)$ for $x \in P_{E}^{m}$. We define a base change lift $\theta_{L}$ of $\theta$ to $L^{\times}$by setting $\theta_{L}=\theta \circ n_{E_{L} \mid E}$. Then $\theta_{L}(1+x)=\psi_{L}\left(\operatorname{tr}_{E_{L} \mid L} \gamma x\right)$ for $x \in$ $P_{E_{L}}^{m}$. (Recall that $m=[(n+2) / 2]$.) The base change lift $\rho_{L}$ of $\left.\rho\right|_{H^{1}}$ to $H_{L}^{1}=$ $L^{\times}\left(1+P_{E_{L}}\right) K_{L}^{m}$ is defined by

$$
\rho_{L}(h \cdot g)=\theta_{L}(h) \psi_{L}(\operatorname{Tr} \gamma(g-1)) \quad \text { for } \quad h \in L^{\times}\left(1+P_{E_{L}}\right), \quad g \in K_{L}^{m} .
$$

When $n+1=2 m$, we define the base change $\kappa_{L}$ of $\left.\kappa\right|_{B^{1}}$ to $B_{L}^{1}$ by

$$
\kappa_{L}=\operatorname{Ind}_{H_{L}^{1}}^{B_{1}^{1}} \rho_{L}
$$

When $n+1=2 m-1$, there exists an irreducible representation $\eta_{L}$ of $J_{L}^{1}=$ $L^{\times}\left(1+P_{E_{L}}\right) K_{L}^{m-1}$ such that

$$
\operatorname{Ind}_{H_{L}^{1}}^{J_{1}^{1}} \rho_{L}=q^{2} \eta_{L}
$$

Then the base change lift $\kappa_{L}$ of $\left.\kappa_{\theta}\right|_{B^{1}}$ to $B_{L}^{1}$ is given by

$$
\kappa_{L}=\operatorname{Ind}_{J_{L}^{1}}^{B_{L}^{1}} \eta_{L}
$$

By virtue of $\theta_{L} \circ \tau=\theta_{L}$, we have $\rho_{L} \circ \tau=\rho_{L}$. Thus we can define an extension $\tilde{\rho}_{L}$ of $\rho_{L}$ to $H_{L}^{1} \rtimes\langle\tau\rangle$ by

$$
\tilde{\rho}_{L}(x \rtimes \tau)=\rho_{L}(x) \quad \text { for } x \in H_{L}^{1} .
$$

Similarly we can extend $\eta_{L}$ to $J_{L}^{1} \rtimes\langle\tau\rangle$ since $\eta_{L} \circ \tau \simeq \eta_{L}$.

Now we apply the result of Bushnell and Henniart (4]) to our case and get the character relation of $\kappa_{L}$ and $\tilde{\kappa}_{L}$.

Lemma 3.2. There exists an extension $\tilde{\eta}_{L}$ of $\eta_{L}$ to $J_{L}^{1} \rtimes\langle\tau\rangle$ such that

$$
\chi_{\tilde{\eta}_{L}}(x \rtimes \tau)=\chi_{\eta}\left(n_{E_{L} \mid E}(x)\right) \quad \text { for } x \in E_{L}^{\times}
$$

Proof. Let $\tilde{\eta}$ be an extension of $\eta_{L}$ to $J_{L}^{1} \rtimes\langle\tau\rangle$. It follows from Proposition (12.8) in [4] that

$$
\chi_{\tilde{\eta}}(x \rtimes \tau)=\frac{\chi_{\tilde{\eta}}(\tau)}{q} \chi_{\eta}\left(n_{E_{L} \mid E}(x)\right) .
$$

Applying Lemma (13.1) Proposition (ii) to our case, we have

$$
\left|\chi_{\tilde{\eta}}(\tau)\right|=\left.\left.\right|^{\tau}\left(J_{L}^{1} / H_{L}^{1}\right)\right|^{1 / 2}=q^{2} .
$$

Since $\tau$ is order $2, \chi_{\tilde{\eta}}(\tau)=\overline{\chi_{\tilde{\eta}}}$. Thus we obtain $\chi_{\tilde{\eta}}(\tau)= \pm q$. If $\chi_{\tilde{\eta}}(\tau)=-q$, the other extension of $\eta_{L}$ to $J_{L}^{1} \rtimes\langle\tau\rangle$ satisfies the desired equation.

Using Frobenius's Formula and Corollary (12.19) in [4, we see that the following result holds.

Proposition 3.3. Let $x \in L^{\times}\left(1+P_{E_{L}}\right)$.

1. When $n+1=2 m$,

$$
\chi_{\kappa}\left(n_{E_{L} \mid E}(x)\right)=\sum_{\substack{a \in H_{L}^{1} \backslash B_{L}^{1} \\ a x^{\tau} a^{-1} \in H_{L}^{1}}} \rho_{L}\left(a x^{\tau} a^{-1}\right) .
$$


2. When $n+1=2 m-1$,

$$
\begin{aligned}
\chi_{\kappa}\left(n_{E_{L} \mid E}(x)\right) & =\sum_{\substack{a \in J_{L}^{1} \backslash B_{L}^{1} \\
a x^{\tau} a^{-1} \in J_{L}^{1}}} \chi_{\eta_{L}}\left(a x^{\tau} a^{-1} \rtimes \tau\right) \\
& =q \sum_{\substack{a \in J_{L}^{1} \backslash B_{L}^{1} \\
a x^{\tau} a^{-1} \in H_{L}^{1}}} \rho_{L}\left(a x^{\tau} a^{-1}\right) .
\end{aligned}
$$

We recall that $J_{L}^{1}=L^{\times}\left(1+P_{E_{L}}\right) K_{L}^{m^{\prime}}$ for $m^{\prime}=[(n+1) / 2]$. Since $m^{\prime}=m$ if $n+1=2 m$, we get $J_{L}^{1}=H_{L}^{1}$ if $n+1=2 m$. Thus we have only to consider the coset $J_{L}^{1} \backslash B_{L}^{1}$.

We proceed in the same way as section 2 . Set $\xi=\operatorname{diag}\left(1, \zeta^{2}, \zeta\right) \in \mathrm{M}_{3}(L)$. Then $\xi$ satisfies $\xi^{3}=1,{ }^{\tau} \xi=\xi^{2}$,

$$
\xi x \xi^{-1}={ }^{\sigma} x \quad \text { for any } x \in E_{L}
$$

and

$$
\begin{array}{ccccccc}
\mathrm{M}_{3}(L) & = & E_{L} & \oplus & E_{L} \xi & \oplus & E_{L} \xi^{2} \\
A_{L}^{0} & = & \mathcal{O}_{E_{L}} & \oplus & \mathcal{O}_{E_{L}} \xi & \oplus & \mathcal{O}_{E_{L}} \xi^{2} \\
A_{L}^{1} & = & P_{E_{L}} & \oplus & P_{E_{L}} \xi & \oplus & P_{E_{L}} \xi^{2} \\
A_{L}^{2} & = & P_{E_{L}}^{2} & \oplus & P_{E_{L}}^{2} \xi & \oplus & P_{E_{L}}^{2} \xi^{2}
\end{array}
$$

By Lemma 2.2 the set

$$
\begin{aligned}
& \left\{1+\beta_{1} \xi+\beta_{2} \xi^{2} \mid \beta_{i} \in \mathcal{O}_{E_{L}} / P_{E_{L}}^{m^{\prime}}, \operatorname{det}\left(1+\beta_{1} \xi+\beta_{2} \xi^{2}\right) \in \mathcal{O}_{E_{L}}^{\times}\right\} \\
\cup & \left\{\left(1+\beta_{1} \xi+\beta_{2} \xi^{2}\right) \xi \mid \beta_{1} \in P_{E_{L}} / P_{E_{L}}^{m^{\prime}}, \beta_{2} \in P_{E_{L}} / P_{E_{L}}^{m^{\prime}}\right\} \\
\cup & \left\{\left(1+\beta_{1} \xi+\beta_{2} \xi^{2}\right) \xi^{2} \mid \beta_{1} \in P_{E_{L}} / P_{E_{L}}^{m^{\prime}}, \beta_{2} \in \mathcal{O}_{E_{L}} / P_{E_{L}}^{m^{\prime}}, \operatorname{det}\left(1+\beta_{2} \xi^{2}\right) \in \mathcal{O}_{E_{L}}^{\times}\right\}
\end{aligned}
$$

gives a complete set of representatives of $J_{L} \backslash B_{L}$. If $y \in U_{i}^{*}$, then there exists an element $x \in L^{\times}\left(1+P_{E_{L}}^{i}\right)-L^{\times}\left(1+\left(P_{E_{L}}^{i} \cap \operatorname{Ker}_{\operatorname{tr}_{E_{L} \mid E}}\right)+P_{E_{L}}^{i+1}\right)$ such that $n_{E_{L} \mid E}(x)=y$. Thus it suffices to calculate the right-hand sides of (3.1) and (3.2) for $x \in L^{\times}\left(1+P_{E_{L}}^{i}\right)-L^{\times}\left(1+\left(P_{E_{L}}^{i} \cap \operatorname{Ker}_{\operatorname{tr}_{E_{L}} \mid E}\right)+P_{E_{L}}^{i+1}\right)$ with $i \geq 0$. The case $n_{E_{L} \mid E}(x) \in E^{\times}-U_{1}$ is treated in Lemma3.8. Now we assume $i \geq 1$.

Lemma 3.4. Let $x \in L^{\times}\left(1+P_{E_{L}}^{i}\right)-L^{\times}\left(1+\left(P_{E_{L}}^{i} \cap \operatorname{Ker}_{\operatorname{tr}_{E_{L}} \mid E}\right)+P_{E_{L}}^{i+1}\right)$ for $i \geq 1$ and $a \in B_{L}$.

1. $a x^{\tau} a^{-1} \in H_{L}$ if and only if $H_{L} a=H_{L}{ }^{\tau} a$ and $H_{L} a=H_{L} a x$.

2. When $a=1+\beta_{1} \xi+\beta_{2} \xi^{2}$ for $\beta_{1}, \beta_{2} \in \mathcal{O}_{E_{L}}$, ax ${ }^{\tau} a^{-1} \in H_{L}$ is equivalent to $\beta_{1}, \beta_{2} \in P_{E_{L}}^{m^{\prime}-i}$ and $\beta_{2}={ }^{\tau} \beta_{1} \bmod P_{E_{L}}^{m^{\prime}}$.

3. When $a=\left(1+\beta_{1} \xi+\beta_{2} \xi^{2}\right) \xi^{2}$ for $\beta_{1} \in P_{E_{L}}$ and $\beta_{2} \in \mathcal{O}_{E_{L}}$, ax ${ }^{\tau} a^{-1} \in H_{L}$ is equivalent to $i \geq m^{\prime}, n_{E_{L} \mid E}\left(\beta_{2}\right) \in 1+P_{E}^{m^{\prime}}$ and $\beta_{1}=\left({ }^{\tau} \beta_{2}\right)^{-1 \tau} \beta_{1} \bmod P_{E_{L}}^{m^{\prime}}$.

4. When $a=\left(1+\beta_{1} \xi+\beta_{2} \xi^{2}\right) \xi$ for $\beta_{1}, \beta_{2} \in P_{E_{L}}$, ax ${ }^{\tau} a^{-1} \notin H_{L}$.

Proof. First we prove 2. If $a x^{\tau} a^{-1} \in H_{L}$, then there exist $\gamma_{0} \in \mathcal{O}_{E_{L}}^{\times}$and $\gamma_{1}, \gamma_{2} \in$ $P_{E_{L}}^{m^{\prime}}$ such that

$$
\left(1+\beta_{1} \xi+\beta_{2} \xi^{2}\right) x=\gamma_{0}\left(1+\gamma_{1} \xi+\gamma_{2} \xi^{2}\right)\left(1+{ }^{\tau} \beta_{2} \xi+{ }^{\tau} \beta_{1} \xi^{2}\right) .
$$


Therefore,

$$
\begin{aligned}
x & =\gamma_{0}\left(1+\gamma_{1}{ }^{\sigma \tau} \beta_{1}+\gamma_{2}{ }^{\sigma^{2} \tau} \beta_{2}\right), \\
\beta_{1}{ }^{\sigma} x & =\gamma_{0}\left(\gamma_{1}+{ }^{\tau} \beta_{2}+\gamma_{2}{ }^{\sigma^{2} \tau} \beta_{1}\right), \\
\beta_{2}{ }^{2} x & =\gamma_{0}\left(\gamma_{2}+{ }^{\tau} \beta_{1}+\gamma_{1}{ }^{\sigma \tau} \beta_{2}\right) .
\end{aligned}
$$

By multiplying the first equation on the right successively by ${ }^{\tau} \beta_{2}$ and ${ }^{\tau} \beta_{1}$ and using the fact that $\gamma_{1}, \gamma_{2} \in P_{E_{L}}^{m^{\prime}}$, we see that

$$
\begin{gathered}
\beta_{1}{ }^{\sigma} x=x^{\tau} \beta_{2} \bmod P_{E_{L}}^{m^{\prime}}, \\
\beta_{2}{ }^{\sigma^{2}} x=x^{\tau} \beta_{1} \bmod P_{E_{L}}^{m^{\prime}} .
\end{gathered}
$$

Eliminating $\beta_{2}$ from these two equations and using the fact that $\tau \sigma^{2}=\sigma \tau$, we obtain

$$
\beta_{1}=n_{E_{L} \mid E}(x)^{\sigma} n_{E_{L} \mid E}(x)^{-1} \beta_{1} \bmod P_{E_{L}}^{m^{\prime}} .
$$

Since $n_{E_{L} \mid E}(x) \in U_{i}^{*}$, we have $v_{E}\left(n_{E_{L} \mid E}(x)^{\sigma} n_{E_{L} \mid E}(x)^{-1}-1\right)=i$, and so $\beta_{1} \in$ $P_{E_{L}}^{m^{\prime}-i}$; similarly, $\beta_{2} \in P_{E_{L}}^{m^{\prime}-i}$. Since $x^{\sigma} x^{-1} \in 1+P_{E_{L}}^{i}$, it follows that $\beta_{1}={ }^{\tau} \beta_{2} \bmod$ $P_{E_{L}}^{m^{\prime}}$. Then it follows from Lemma 2.2 that ${ }^{\tau} a \equiv a \bmod H_{L}$. Next we prove 3. If $a x^{\tau} a^{-1} \in H_{L}$, then there exist $\gamma_{0} \in \mathcal{O}_{E_{L}}^{\times}$and $\gamma_{1}, \gamma_{2} \in P_{E_{L}}^{m^{\prime}}$ such that

$$
\left(\xi^{2}+\beta_{1}+\beta_{2} \xi\right) x=\gamma_{0}\left(1+\gamma_{1} \xi+\gamma_{2} \xi^{2}\right)\left(\xi+{ }^{\tau} \beta_{1}+{ }^{\tau} \beta_{2} \xi^{2}\right) .
$$

From ${ }^{\tau} \xi=\xi^{2}$ and ${ }^{\tau} \xi^{2}=\xi$, this implies that

$$
\begin{aligned}
\sigma^{2} x & =\gamma_{0}\left(\gamma_{1}+\gamma_{2}{ }^{\sigma^{2} \tau} \beta_{1}+{ }^{\tau} \beta_{2}\right), \\
\beta_{1} x & =\gamma_{0}\left({ }^{\tau} \beta_{1}+\gamma_{1}{ }^{\sigma \tau} \beta_{2}+\gamma_{2}\right), \\
\beta_{2}{ }^{\sigma} x & =\gamma_{0}\left(1+\gamma_{1}{ }^{\sigma \tau} \beta_{1}+\gamma_{2}{ }^{\sigma^{2} \tau} \beta_{2}\right) .
\end{aligned}
$$

In the same way as the proof of 1 , we can show that

$$
\sigma^{2} x=n_{E_{L} \mid E}\left(\beta_{2}\right)^{\sigma} x \quad \bmod P_{E_{L}}^{m^{\prime}} \quad \text { and } \quad \beta_{1} x={ }^{\sigma} x \beta_{2}{ }^{\tau} \beta_{1} \bmod P_{E_{L}}^{m^{\prime}} \text {. }
$$

Since $n_{E_{L} \mid E}\left(\beta_{2}\right)$ is $\tau$-invariant, we have ${ }^{\tau \sigma}\left({ }^{\sigma} x x^{-1}\right)={ }^{\sigma}\left({ }^{\sigma} x x^{-1}\right) \bmod P_{E_{L}}^{m^{\prime}}$. Using $\tau \sigma^{2}=\sigma \tau$ and $\tau \sigma=\sigma^{2} \tau$, we get ${ }^{\sigma}\left({ }^{\tau} x x\right)=\sigma^{2}\left({ }^{\tau} x x\right) \bmod P_{E_{L}}^{m^{\prime}}$. This implies ${ }^{\sigma} n_{E_{L} \mid E}(x)=n_{E_{L} \mid E}(x) \bmod P_{E_{L}}^{m^{\prime}}$. Therefore we obtain $n_{E_{L} \mid E}(x) \in F^{\times}\left(1+P_{E}^{m^{\prime}}\right)$. By the assumption $x \in L^{\times}\left(1+P_{E_{L}}^{i}\right)-L^{\times}\left(1+\left(P_{E_{L}}^{i} \cap \operatorname{Ker} \operatorname{tr}_{E_{L} \mid E}\right)+P_{E_{L}}^{i+1}\right)$, we get $i \geq m^{\prime}$ and ${ }^{\sigma} x x^{-1} \in 1+P_{E_{L}}^{m^{\prime}}$. It follows from (3.5) that $n_{E_{L} \mid E}\left(\beta_{2}\right) \in 1+P_{E_{L}}^{m^{\prime}}$ and $\beta_{1}=\left({ }^{\tau} \beta_{2}\right)^{-1 \tau} \beta_{1} \bmod P_{E_{L}}^{m^{\prime}}$. This implies $H_{L} a=H_{L}{ }^{\tau} a$ by Lemma 2.2. Finally we prove 4. By the same argument as above, $a x{ }^{\tau} a^{-1} \in H_{L}$ implies $\beta_{2} x \equiv{ }^{\sigma^{2}} x \beta_{1}{ }^{\tau} \beta_{2}$ $\bmod P_{E_{L}}^{m^{\prime}}$. Since $\beta_{1} \in P_{E_{L}}, v_{E_{L}}\left(\sigma^{2} x \beta_{1}{ }^{\tau} \beta_{2}\right) \geq v_{E_{L}}\left(\beta_{2}\right)+1$. This is impossible. As for 1 , it follows from (3.4) and the proofs of 2,3 and 4 .

Lemma 3.5. 1. Let $x \in \mathcal{O}_{E_{L}}^{\times}$satisfy $n_{E_{L} \mid E}(x) \in U_{i}^{*}(i>0)$ and $a=1+\beta \xi+{ }^{\tau} \beta \xi^{2}$ for $v_{E_{L}}(\beta) \geq \max \left(0, m^{\prime}-i\right)$. Then

$$
\rho_{L}\left(a x^{\tau} a^{-1} x^{-1}\right)=\psi_{E}\left(S(\beta) \operatorname{tr}_{E_{L} \mid E}(x-1)\right),
$$


where

$$
S(\beta)=\frac{\operatorname{tr}_{E_{L} \mid E}\left(\left(\gamma-{ }^{\sigma} \gamma\right)\left(n_{\left.E_{L}\right|^{\sigma^{2} E}}(\beta)-n_{E_{L} \mid L}\left({ }^{\tau} \beta\right)\right)\right)}{1+\operatorname{tr}_{L \mid F}\left(n_{E_{L} \mid L}(\beta)\right)-\operatorname{tr}_{\sigma^{2} E \mid F}\left(n_{\left.E_{L}\right|^{2} E^{2}}(\beta)\right)}
$$

2. Let $\beta_{1} \in P_{E_{L}}, \beta_{2} \in \mathcal{O}_{E_{L}}$ satisfy $n_{E_{L} \mid E}\left(\beta_{2}\right)=1$ and $\beta_{1}=\left({ }^{\tau} \beta_{2}\right)^{-1 \tau} \beta_{1}$. Put $a=\left(1+\beta_{1} \xi+\beta_{2} \xi^{2}\right) \xi^{2}$. For $n_{E_{L} \mid E}(x) \in U_{m^{\prime}}$,

$$
\rho_{L}\left(a x^{\tau} a^{-1} x^{-1}\right)=\psi_{E}\left(T\left(\beta_{1}, \beta_{2}\right) \operatorname{tr}_{E_{L} \mid E}(x-1)\right),
$$

where

$$
T\left(\beta_{1}, \beta_{2}\right)=\frac{\left(\sigma_{\gamma}-\gamma\right)\left(1-\sigma^{2} \beta_{1} \beta_{2}\right)+\left(\sigma^{2} \gamma-\gamma\right)\left(n_{E_{L} \mid L}\left(\beta_{2}\right)-{ }^{\sigma} \beta_{1} \sigma^{2} \beta_{2}\right)}{1+n_{E_{L} \mid L}\left(\beta_{2}\right)+n_{E_{L} \mid L}\left(\beta_{1}\right)-\operatorname{tr}_{E_{L} \mid L}\left(\beta_{1}{ }^{\sigma} \beta_{2}\right)} .
$$

Proof. By Lemma 3.4 ${ }^{\tau} a a^{-1} \in H_{L}$. This implies $\rho_{L}\left(a x^{\tau} a^{-1} x^{-1}\right)=\rho_{L}\left(a x a^{-1} x^{-1}\right)$, since $\rho_{L}\left({ }^{\tau} a a^{-1}\right)=1$. Thus we can prove the first part in the same way as Lemma [2.6. Now we prove the case $a=\left(1+\beta_{1} \xi+\beta_{2} \xi^{2}\right) \xi^{2}$. Put $x=1+y$ for $y \in P_{E_{L}}^{m^{\prime}}$. By Lemma 2.5

$$
=1+\frac{\left(\beta_{1} y \xi+\beta_{2}{ }^{\sigma} y \xi^{2}+{ }^{\sigma^{2}} y\right)\left(1-{ }^{\sigma} \beta_{1} \sigma^{2} \beta_{2}+\left(\beta_{2}{ }^{\sigma^{2}} \beta_{2}-\beta_{1}\right) \xi+\left(\beta_{1}{ }^{\sigma} \beta_{1}-\beta_{2}\right) \xi^{2}\right)}{\operatorname{det}(a)} .
$$

Thus we have

$$
\begin{aligned}
& \rho_{L}\left(a g a^{-1} g^{-1}\right) \\
& =\psi_{E_{L}}\left(\frac{\left({ }^{\sigma} \gamma-\gamma\right)\left(1-\sigma^{2} \beta_{1} \beta_{2}\right)+\left(\sigma^{2} \gamma-\gamma\right)\left(n_{E_{L} \mid L}\left(\beta_{2}\right)-{ }^{\sigma} \beta_{1} \sigma^{2} \beta_{2}\right)}{1+n_{E_{L} \mid L}\left(\beta_{2}\right)+n_{E_{L} \mid L}\left(\beta_{1}\right)-\operatorname{tr}_{E_{L} \mid L}\left(\beta_{1} \sigma \beta_{2}\right)}(x-1)\right) .
\end{aligned}
$$

Therefore it suffices to say that $T\left(\beta_{1}, \beta_{2}\right)$ is $\tau$-invariant. It follows from ${ }^{\tau} \beta_{1}={ }^{\tau} \beta_{2} \beta_{1}$, ${ }^{\tau} \beta_{2} \beta_{2}=1$ and $\gamma \in E$ that

$$
\begin{aligned}
\tau_{T}\left(\beta_{1}, \beta_{2}\right) & =\frac{\left({ }^{2} \gamma-\gamma\right)\left(1-{ }^{\sigma} \beta_{1} \sigma^{2} \beta_{2} / n_{E_{L} \mid L}\left(\beta_{2}\right)\right)+\left({ }^{\sigma} \gamma-\gamma\right)\left(1-{ }^{2} \beta_{1} \beta_{2}\right) / n_{E_{L} \mid E}\left(\beta_{2}\right)}{\left(n_{E_{L} \mid L}\left(\beta_{2}\right)+n_{E_{L} \mid L}\left(\beta_{1}\right)+1-\operatorname{tr}_{E_{L} \mid L}\left(\beta_{1} \beta_{2}\right)\right) / n_{E_{L} \mid E}\left(\beta_{2}\right)} \\
& =T\left(\beta_{1}, \beta_{2}\right) .
\end{aligned}
$$

Lemma 3.6. Put $E^{0}=\operatorname{Ker}_{\operatorname{tr}}$ and $C^{\prime}(\mu)=q^{m^{\prime}-\mu-1+\left[\left(m^{\prime}-\mu-n\right) / 3\right]+[(n-2 \mu-1) / 3]}$.

1. Let $\mu$ be a positive integer. If $n \not \equiv 2 \mu \bmod 3($ resp. $n \equiv 2 \mu \bmod 3)$, then the map $x \mapsto S(x)$ induces a surjection from $\varpi_{E}^{\mu} \mathcal{O}_{E_{L}}^{\times} / 1+P_{E_{L}}^{m^{\prime}-\mu}$ to $\left(P_{E}^{2 \mu-n}-\right.$ $\left.P_{E}^{2 \mu-n+1}\right) \cap E^{0} / P_{E}^{m^{\prime}+\mu-n} \cap E^{0}$ (resp. $\left.P_{E}^{2 \mu-n+1} \cap E^{0} / P_{E}^{m^{\prime}+\mu-n} \cap E^{0}\right)$ and each fiber of the map has $(q+1) C^{\prime}(\mu)$ (resp. $\left.\left(q^{2}-1\right) C^{\prime}(\mu)\right)$ elements.

2. For any $x_{0} \in \mathcal{O}_{E_{L}}^{\times}$such that $1+\operatorname{tr}_{L \mid F}\left(n_{E_{L} \mid L}\left(x_{0}\right)\right)-\operatorname{tr}_{\sigma^{2} E \mid F}\left(n_{\left.E_{L}\right|^{2} E^{2}}\left(x_{0}\right)\right) \notin P_{F}$, the map $x \mapsto S(x)$ induces a surjection from $\left\{x \in \mathcal{O}_{E_{L}}^{\times} / 1+P_{E_{L}}^{m^{\prime}} \mid x \equiv x_{0} \bmod P_{E_{L}}\right\}$ to

$$
\left\{x \in P_{E}^{-n} \cap E^{0} / P_{E}^{m^{\prime}-n} \cap E^{0} \mid x \equiv S\left(x_{0}\right) \quad \bmod P_{E}^{1-n}\right\}
$$

and each fiber of the map has $C^{\prime}(0)$ elements. 
3. Fix $\beta_{2} \in \mathcal{O}_{E_{L}}$ such that $n_{E_{L} \mid E}\left(\beta_{2}\right)=1$. Then the map $x \mapsto T\left(x, \beta_{2}\right)$ induces a surjection from $\left\{x \in P_{E_{L}} \mid{ }^{\tau} x={ }^{\tau} \beta_{2} x\right\}$ to

$$
\left\{x \in P_{E}^{-n} \cap E^{0} / P_{E}^{m^{\prime}-n} \cap E^{0} \mid x \equiv \frac{\sigma_{\gamma}-\gamma+\left(\sigma^{2} \gamma-\gamma\right) n_{E_{L} \mid L}\left(\beta_{2}\right)}{1+n_{E_{L} \mid L}\left(\beta_{2}\right)} \quad \bmod P_{E}^{1-n}\right\}
$$

and each fiber of the map has $q^{\left[\left(m^{\prime}-n\right) / 3\right]+[(n-1) / 3]}$ elements.

Proof. Since

$$
\begin{aligned}
S(\beta) & =\frac{\operatorname{tr}_{E_{L} \mid E}\left(\left(\gamma-{ }^{\sigma} \gamma\right)\left(n_{E_{L} \mid \sigma^{2} E}(\beta)-n_{E_{L} \mid L}\left({ }^{\tau} \beta\right)\right)\right)}{1+\operatorname{tr}_{L \mid F}\left(n_{E_{L} \mid L}(\beta)\right)-\operatorname{tr}_{\sigma^{2} E \mid F}\left(n_{E_{L} \mid \sigma^{2} E}(\beta)\right)} \\
& =\frac{\left(\gamma-{ }^{\sigma} \gamma\right)\left(n_{E_{L} \mid \sigma^{2} E}(\beta)-n_{E_{L} \mid L}\left({ }^{\tau} \beta\right)\right)-\sigma^{2}\left(\left(\gamma-{ }^{\sigma} \gamma\right)\left(n_{E_{L} \mid \sigma_{E}{ }^{2}}(\beta)-n_{E_{L} \mid L}\left({ }^{\tau} \beta\right)\right)\right)}{1+\operatorname{tr}_{L \mid F}\left(n_{E_{L} \mid L}(\beta)\right)-\operatorname{tr}_{\sigma^{2} E \mid F}\left(n_{E_{L} \mid \sigma^{2} E}(\beta)\right)},
\end{aligned}
$$

$S(\beta)$ belongs to $E^{0}$. When $v_{E_{L}}(\beta)=\mu$, we have $S(\beta) \in P_{E}^{2 \mu-n}, P_{E}^{2 \mu-n} \cap E^{0}=$ $P_{E}^{2 \mu-n+1} \cap E^{0}$ if $n \not \equiv 2 \mu \bmod 3$ and

$$
\begin{aligned}
& S(\beta(1+x)) \\
& \equiv S(\beta)+\operatorname{tr}_{E_{L} \mid E}\left(\left(\gamma-{ }^{\sigma} \gamma\right) n_{\left.E_{L}\right|^{\sigma^{2} E}}(\beta) \operatorname{tr}_{\left.E_{L}\right|^{\sigma^{2} E}}(x)\right) \quad \bmod P_{E}^{\mu-n+v_{E_{L}}(x)+1} .
\end{aligned}
$$

for $x \in P_{E_{L}}$. Therefore $x \mapsto S(x)$ induces a well-defined map from

$$
\varpi_{E}^{\mu} \mathcal{O}_{E_{L}}^{\times} /\left(1+P_{E_{L}}^{m^{\prime}-\mu}\right)
$$

to

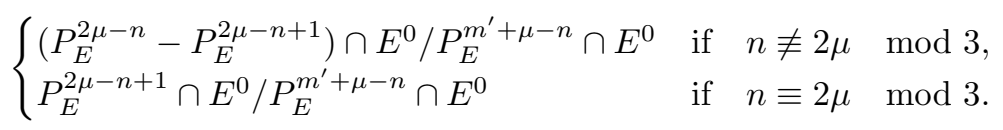

Denote the induced map by $S_{m^{\prime}-\mu}$ and put

$$
c_{i}=\left\{\begin{array}{lll}
(q+1) q^{i-1+\left[\left(2 m^{\prime}-i-n\right) / 3\right]+\left[\left(n-2 m^{\prime}+2 i-1\right) / 3\right]} & n \neq \equiv 2 \mu & \bmod 3 \\
\left(q^{2}-1\right) q^{i-1+\left[\left(2 m^{\prime}-i-n\right) / 3\right]+\left[\left(n-2 m^{\prime}+2 i-1\right) / 3\right]} & n \equiv 2 \mu & \bmod 3 .
\end{array}\right.
$$

First assume $\mu>0$. We prove the assertion by induction on $m^{\prime}-\mu$. Let $m^{\prime}-\mu=1$. When $n \equiv 2 \mu \bmod 3, P_{E}^{2 \mu-n+1} \cap E^{0} / P_{E}^{m^{\prime}+\mu-n}=P_{E}^{2 m^{\prime}-n-1} \cap E^{0} / P_{E}^{2 m^{\prime}-n-1} \cap E^{0}$. Since $\left|\varpi_{E}^{\mu} \mathcal{O}_{E_{L}}^{\times} /\left(1+P_{E_{L}}\right)\right|=q^{2}-1, S_{1}$ is surjective and its fiber has $q^{2}-1$ elements. Now assume $n \not \equiv 2 \mu \bmod 3$. Since

$$
S(x) \equiv\left(\gamma-{ }^{\sigma} \gamma\right) n_{\left.E_{L}\right|^{\sigma^{2} E}}(x)-{ }^{\sigma^{2}}\left(\left(\gamma-{ }^{\sigma} \gamma\right) n_{\left.E_{L}\right|^{\sigma^{2} E}}(x)\right) \quad \bmod P_{E}^{2 \mu-n+1} \cap E^{0}
$$

and $n_{\left.E_{L}\right|^{\sigma^{2} E}}$ induces a surjective homomorphism from $\mathcal{O}_{E_{L}}^{\times} /\left(1+P_{E_{L}}\right)$ to $\mathcal{O}_{\sigma^{2} E}^{\times} /(1+$ $\left.P_{\sigma^{2} E}\right)$, it follows that $S_{1}$ is a surjection and each fiber of $S_{1}$ has $q+1$ elements. Let $m^{\prime}-\mu>1$ and take any element $y \in\left(P_{E}^{2 \mu-n}-P_{E}^{2 \mu-n+1}\right) \cap E^{0}$. By the induction assumption, there exist $c_{m^{\prime}-\mu-1}$ elements $x \in \varpi_{E}^{\mu} \mathcal{O}_{E_{L}}^{\times} / 1+P_{E_{L}}^{m^{\prime}-\mu-1}$ such that $S_{m^{\prime}-\mu-1}(x)=y \bmod P_{E}^{m^{\prime}+\mu-n-1}$. Put $x^{\prime}=x\left(1+x_{1}\right)$ for $x_{1} \in P_{E_{L}}^{m^{\prime}-\mu-1}$. Then

$$
\begin{aligned}
& S\left(x^{\prime}\right) \equiv S(x)+\operatorname{tr}_{E_{L} \mid E}\left(\left(\gamma-{ }^{\sigma} \gamma\right) n_{\left.E_{L}\right|^{\sigma^{2} E}}(x)\left(n_{\left.E_{L}\right|^{\sigma^{2} E}}\left(1+x_{1}\right)-1\right)\right) \\
& \bmod P_{E}^{m^{\prime}+\mu-n} \cap E^{0} \\
& \equiv S(x)+\left(\gamma-{ }^{\sigma} \gamma\right) n_{\left.E_{L}\right|^{\sigma^{2}} E}(x) \operatorname{tr}_{\left.E_{L}\right|^{\sigma^{2} E}} x_{1} \\
& -\sigma^{2}\left(\left(\gamma-{ }^{\sigma} \gamma\right) n_{\left.E_{L}\right|^{\sigma^{2} E}}(x) \operatorname{tr}_{\left.E_{L}\right|^{\sigma^{2} E}} x_{1}\right) \quad \bmod P_{E}^{m^{\prime}+\mu-n} \cap E^{0} .
\end{aligned}
$$


Since $x_{1} \mapsto\left(\gamma-{ }^{\sigma} \gamma\right) n_{\left.E_{L}\right|^{\sigma^{2} E}}(x) \operatorname{tr}_{\left.E_{L}\right|^{\sigma^{2} E}} x_{1}-\sigma^{2}\left(\left(\gamma-{ }^{\sigma} \gamma\right) n_{E_{L} \mid \sigma^{2} E}(x) \operatorname{tr}_{\left.E_{L}\right|^{\sigma^{2} E}} x_{1}\right)$ induces a surjective $k_{F}$ linear map from $P_{E_{L}}^{m^{\prime}-\mu-1} / P_{E_{L}}^{m^{\prime}-\mu}$ to $P_{E}^{m^{\prime}+\mu-n-1} \cap E^{0} /$ $P_{E}^{m^{\prime}+\mu-n} \cap E^{0}$, it follows that $S_{m^{\prime}-\mu}$ is surjective and each fiber of it has

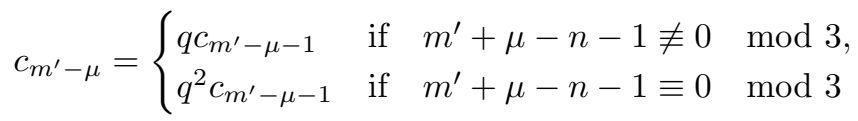

elements. The case $\mu=0$ can be proved in the same way. For the map $x \mapsto T\left(x, \beta_{2}\right)$, we can prove our assertion in the same way as the proof of Lemma 2.7 since

$$
T\left(x, \beta_{2}\right)=\frac{\sigma_{\gamma}-\gamma+\left({ }^{\sigma^{2}} \gamma-\gamma\right) n_{E_{L} \mid L}\left(\beta_{2}\right)}{1+n_{E_{L} \mid L}\left(\beta_{2}\right)}+\frac{\left(\gamma-{ }^{\sigma} \gamma\right)^{\sigma^{2}} x \beta_{2}-{ }^{\sigma^{2}}\left(\left(\gamma-{ }^{\sigma} \gamma\right)^{\sigma^{2}} x \beta_{2}\right)}{1+n_{E_{L} \mid L}\left(\beta_{2}\right)} .
$$

By the above three lemmas, we have the following lemma. See also the proof of Lemma 2.8 and Proposition 2.9.

Lemma 3.7. 1. Let $0<\mu<m$ and $n \not \equiv 2 \mu \bmod 3$. For $x \in \mathcal{O}_{E_{L}}^{\times}$,

$$
\begin{aligned}
\sum_{\substack{a=1+\beta \xi+^{\tau} \beta \xi^{2} \\
\beta \in \varpi_{E}^{\mu} \mathcal{O}_{E_{L}}^{\times} / 1+P_{E_{L}}^{m^{\prime}-\mu}}} \rho_{L}\left(a x^{\tau} a^{-1} x^{-1}\right) \\
=\left\{\begin{array}{lll}
0 & \text { if } n_{E_{L} \mid E}(x) \notin U_{n-2 \mu}, \\
-(q+1) q^{2\left(m^{\prime}-\mu-1\right)} & \text { if } & n_{E_{L} \mid E}(x) \in U_{n-2 \mu}^{*}, \\
\left(q^{2}-1\right) q^{2\left(m^{\prime}-\mu-1\right)} & \text { if } & n_{E_{L} \mid E}(x) \in U_{n+1-2 \mu} .
\end{array}\right.
\end{aligned}
$$

2. Let $0<\mu<m$ and $n \equiv 2 \mu \bmod 3$. For $x \in \mathcal{O}_{E_{L}}^{\times}$,

$$
\begin{aligned}
\sum_{\substack{a=1+\beta \xi+{ }^{\tau} \beta \xi^{2} \\
\beta \in \varpi_{E}^{\mu} \mathcal{O}_{E_{L}}^{\times} / 1+P_{E_{L}}^{m^{\prime}-\mu}}} \rho_{L}\left(a x^{\tau} a^{-1} x^{-1}\right) \\
=\left\{\begin{array}{lll}
0 & \text { if } n_{E_{L} \mid E}(x) \notin U_{n-2 \mu}, \\
\left(q^{2}-1\right) q^{2\left(m^{\prime}-\mu-1\right)} & \text { if } n_{E_{L} \mid E}(x) \in U_{n+1-2 \mu} .
\end{array}\right.
\end{aligned}
$$

3. For $x \in \mathcal{O}_{E_{L}}^{\times}$,

$$
\sum_{\substack{a=1+\beta \xi+^{\tau} \beta \xi^{2} \\ \beta \in \mathcal{O}_{E_{L}}^{\times} /\left(1+P_{E_{L}}^{m^{\prime}}\right)}} \rho_{L}\left(a x^{\tau} a^{-1} x^{-1}\right)=0 \quad \text { if } n_{E_{L} \mid E}(x) \notin U_{n} .
$$

4. Fix $\beta_{2} \in \operatorname{Ker} n_{E_{L} \mid E}$. For $x \in \mathcal{O}_{E_{L}}^{\times}$,

$$
\sum_{\substack{a=\left(1+\beta_{1} \xi+^{\tau} \beta \xi^{2}\right) \xi^{2} \\ \in\left\{y \in P_{E_{L}} / P_{E_{L}}^{m^{\prime}}||^{\tau} y={ }^{\tau} \beta_{2} y\right\}}} \rho_{L}\left(a x^{\tau} a^{-1} x^{-1}\right)=0 \quad \text { if } n_{E_{L} \mid E}(x) \notin U_{n} .
$$

By this lemma, we can calculate $\chi_{\kappa_{\theta}}$ on $\mathcal{O}_{E}^{\times}-U_{n}$. On $U_{n}^{*}$, it is already calculated in Lemma 2.10. By Theorem 1.8, it gives $\chi_{\pi_{\theta}}$ on $\mathcal{O}_{E}^{\times}-U_{n+1}$. On $U_{n+1}$, it is given in the proof of Theorem 2.13 It remains to calculate $\chi_{\kappa_{\theta}}$ on $E^{\times}-U_{1}$. 
Lemma 3.8. For $x \in E^{\times}-U_{1}$,

$$
\chi_{\kappa_{\theta}}(x)=(-1)^{n+1} \theta(x) .
$$

Proof. By (1.3) and (1.5), it suffices to show that

$$
\chi_{\operatorname{Ind}_{H}^{B} \rho_{\theta}}(x)=\theta(x) .
$$

Since

$$
\chi_{\operatorname{Ind}_{H}^{B} \rho_{\theta}}(x)=\sum_{a \in H \backslash B} \rho_{\theta}\left(a x a^{-1}\right),
$$

we have only to show that if $a x a^{-1} \in H$ for $a \in B$, then $a \in H$.

Let $R$ be a natural ring morphism from $A^{0}$ to $k_{F}^{3}$ by the identification $A^{0} / A^{1}$ with $k_{F}^{3}$. We note that if $R(a)=\left(\alpha_{0}, \alpha_{1}, \alpha_{2}\right)$, then $R\left(\varpi_{E} a \varpi_{E}^{-1}\right)=\left(\alpha_{1}, \alpha_{2}, \alpha_{0}\right)$ and if $a \in E$, then $\alpha_{0}=\alpha_{1}=\alpha_{2}$. We may assume $a \in A^{0}$. Let $R(a)=\left(\alpha_{0}, \alpha_{1}, \alpha_{2}\right)$ and $x=\varpi_{E}^{i} x_{0}$ for $x_{0} \in \mathcal{O}_{E}^{\times}$. Since $x \notin F^{\times}\left(1+P_{E}\right)$,

$$
R\left(\operatorname{axa}^{-1} x^{-1}\right)=\left\{\begin{array}{lll}
\left(\alpha_{0} \alpha_{1}^{-1}, \alpha_{1} \alpha_{2}^{-1}, \alpha_{2} \alpha_{0}^{-1}\right) & \text { if } i \equiv 1 \quad \bmod 3, \\
\left(\alpha_{0} \alpha_{2}^{-1}, \alpha_{1} \alpha_{0}^{-1}, \alpha_{2} \alpha_{1}^{-1}\right) & \text { if } \quad i \equiv 2 \quad \bmod 3 .
\end{array}\right.
$$

Therefore $a x a^{-1} x^{-1} \in E^{\times} K^{1}$ implies $\alpha_{0} \alpha_{1}^{-1}=\alpha_{1} \alpha_{2}^{-1}=\alpha_{2} \alpha_{0}^{-1}$ or $\alpha_{0} \alpha_{2}^{-1}=$ $\alpha_{1} \alpha_{0}^{-1}=\alpha_{2} \alpha_{1}^{-1}$. In any case, $\alpha_{0}=\alpha_{1}=\alpha_{2}$, since $k_{E}=k_{F}$ has no cube root of unity. Thus $a \in E^{\times} K^{1}$. Now we may assume $a-1 \in A^{j}-\left(P_{E}^{j}+A_{E}^{j+1}\right)$ for $j \geq 1$. Put $a-1=\varpi_{E}^{j} a_{0}$ and $R\left(a_{0}\right)=\left(\beta_{0}, \beta_{1}, \beta_{2}\right)$. Since

$$
\begin{aligned}
a x a^{-1} x^{-1} & =1+(a-1)-x(a-1) x^{-1} \bmod A^{j+1} \\
& =1+\varpi_{E}^{j}\left(a_{0}-x a_{0} x^{-1}\right) \bmod A^{j+1},
\end{aligned}
$$

$R\left(a_{0}-x a_{0} x^{-1}\right)=\left(\beta_{0}-\beta_{1}, \beta_{1}-\beta_{2}, \beta_{2}-\beta_{0}\right)$ or $\left(\beta_{0}-\beta_{2}, \beta_{1}-\beta_{0}, \beta_{2}-\beta_{1}\right)$. Therefore $a x a^{-1} x^{-1} \in E^{\times} K^{j+1}$ contradicts $a-1 \in A^{j}-\left(P_{E}^{j}+A^{j+1}\right)$. This implies $a \in E^{\times} K^{m}$ if $a x a^{-1} x^{-1} \in E^{\times} K^{m}$.

Now we can state the character formula of $\pi_{\theta}$ for the non-Galois case. We remark that there is no ramified extension of $F$ with degree 3 which is not isomorphic to E.

Theorem 3.9. Let $q \equiv 2 \bmod 3$, and let the other notation be as in Theorem 2.13. Let $x$ be a regular elliptic element in $\mathrm{GL}_{3}(F)$.

1. If $F(x) \mid F$ is unramified, then

\begin{tabular}{|c|c|}
\hline$x$ & $\chi_{\pi_{\theta}}(x)$ \\
\hline$x \notin F^{\times}\left(1+P_{F(x)}^{n+1}\right)$ & 0 \\
\hline$c(1+y)\left(c \in F^{\times}, y \in P_{F(x)}^{n+1}\right)$ & $q^{n-1}\left(q^{2}+q+1\right) \theta(c)$ \\
\hline
\end{tabular}

2. If $x \in E$, then

\begin{tabular}{|c|c|}
\hline$x$ & $\chi_{\pi_{\theta}}(x)$ \\
\hline$x \in E^{\times}-U_{0}$ & $(-1)^{n+1} \theta(x)$ \\
\hline$x \in U_{j}^{*} \quad(1 \leq j \leq n-1)$ & $(-1)^{n+1}(-q)^{j} \theta(x)$ \\
\hline$c(1+y) \in U_{n}^{*}\left(c \in F^{\times}, y \in \varpi_{E}^{n} \mathcal{O}_{E}^{\times}\right)$ & $q^{n-1} \theta(c) \operatorname{Kl}(\gamma y)$ \\
\hline$x \in U_{n+1}$ & $q^{n-1}\left(q^{2}+q+1\right) \theta(x)$ \\
\hline
\end{tabular}

where the Kloosterman sum $\mathrm{Kl}(a)$ is defined by (2.17). 
Proof. The character formula for the unramified case is obtained in the same way as the Galois case. By Proposition 3.3, (3.4), Lemma 3.4, Lemma 3.7 and Lemma 3.8 we get for $x \in E^{\times}-U_{n+1}$,

$$
\chi_{\kappa \theta}(x)= \begin{cases}(-1)^{n+1} \theta(x) & \text { if } \quad x \in E^{\times}-U_{0}, \\ (-1)^{n+1}(-q)^{j} \theta(x) & \text { if } \quad x \in U_{j}^{*} \quad(1 \leq j \leq n-1) .\end{cases}
$$

On $U_{n}^{*}$, this is obtained by Lemma 2.10. By Theorem 1.8 ,

$$
\chi_{\pi_{\theta}}(x)=\chi_{\kappa_{\theta}}(x) \text { for } x \in E^{\times}-U_{n+1} .
$$

On $U_{n+1}$, the proof of Theorem 2.13 holds for the non-Galois case. Thus we get the character formula for $\pi_{\theta}$ on $E^{\times}$.

\section{ACKNowledgement}

The author would like to express his sincere gratitude to the referee for much helpful advice.

\section{REFERENCES}

[1] J. Arthur and L. Clozel, Simple algebras, base change, and the advanced theory of the trace formula, Annals of Math. Studies 120, Princeton Univ. Press, 1989. MR 90m:22041

[2] A. Badulescu, Correspondance entre $\mathrm{GL}_{n}$ et ses formes intérieures en caractéristique positive, Thèse, Université de Paris-sud, Centre d'Orsay, 1999.

[3] C. J. Bushnell and A. Fröhlich, Gauss Sums and $p$-adic Division Algebras, Lecture Notes in Math. 987, Springer, Berlin, 1983. MR 84m:12017

[4] C. J. Bushnell and G. Henniart, Local Tame Lifting For GL(N) I: Simple characters, Inst. Hautes Études Sci. Publ. Math. No. 83 (1996), 105-233. MR 98m:11129

[5] H. Carayol, Représentations cuspidales du groupe linéaire Ann. Scient. École Norm. Sup. 17 (1984), 191-226. MR 86f:22019

[6] L. Corwin and R. Howe, Computing characters of tamely ramified p-adic division algebras, Pac. J. Math. 73 (1977), 461-477. MR 58:11238

[7] L. Corwin, A. Moy and P. J. Sally, Jr., Supercuspidal Character Formulas for $\mathrm{GL}_{l}$, Contemporary Mathematics 191, AMS, 1995, pp. 1-11. MR 96m:22037

[8] P. Deligne, D. Kazhdan, and M.-F. Vignéras, Représentations des algèbres centrales simples p-adiques, in: Représentations des Groupes Réductifs sur un Corps Local, Herman, Paris, 1984, pp. 33-117. MR 86h:11044

[9] R. Godement and H. Jacquet, Zeta functions of simple algebras, Lecture Notes in Math. 260, Springer, Berlin, 1972. MR 49:7241

[10] G. Henniart, Correspondance de Jaquet-Langlands explicite $I$ : le cas modéré de degré premier, in: Séminaire de Théorie des Nombres, Paris 1990-91, Progress in Math. 108, Birkhäuser, Basel, 1993, pp. 85-114. MR 95d:11064

[11] R. Howe, Kirillov theory for compact p-adic groups, Pac. J. Math. 73, (1977), 365-381. MR 58:28314

$[12] \longrightarrow$, Tamely ramified supercuspidal representations of $\mathrm{GL}_{n}(F)$, Pac. J. Math. 73 (1977), 437-460. MR 58:11241

[13] H. Hijikata, H. Saito, and M. Yamauchi, Representations of quaternion algebras over local fields and trace formula of Hecke operators, J. Number Theory 43 (1993), 123-167. MR 94c:11126

[14] H. Jacquet, I. Piatetski-Shapiro and J. Shalika, Automorphic Forms on GL(3) II, Annals of Math. 109 (1979), 213-258. MR 80i:10034b

[15] P. Kutzko, Character formulas for supercuspidal representations of $\mathrm{GL}_{l}, l$ a prime, Amer. J. Math. 109 (1987), 201-222. MR 88k:22003

[16] A. Moy, Local constant and the tame Langlands correspondence, Amer. J. Math. 108 (1986), 863-930. MR 88b:11081

[17] F. Murnaghan, Asymptotic behaviour of supercuspidal characters of p-adic $\mathrm{GL}_{3}$ and $\mathrm{GL}_{4}$ : The generic unramified case, Pacific J. Math. 148, (1991), 107-130. MR 92a:22023 
[18] J. Rogawski, Representations of $\mathrm{GL}(n)$ and division algebras over p-adic field, Duke Math. J. 50 (1983), 161-196. MR 84j:12018

[19] T. Takahashi, Characters of cuspidal unramified series for central simple algebras of prime degree, J. Math. Kyoto Univ. 32-4 (1992), 873-888. MR 94e:11124

[20] Character formula for representations of local quaternion algebras (Wildly ramified case), J. Math. Kyoto Univ. 36-1 (1996), 151-197. MR 97f:11096

[21] _ On the irreducible very cuspidal representations II, J. Math. Kyoto Univ. 36-4 (1996), 889-910. MR 98k:22075

[22] J.-P. Serre, Linear Representations of Finite Groups, Springer Verlag, New-York, 1977. MR 56:8675

Department of Mathematics and Information, College of Integrated Arts and Sciences, Osaka Prefecture University, 1-1 Gakuen-cho Sakai, 599-8531, Japan

E-mail address: takahasi@mi.cias.osakafu-u.ac.jp 